\title{
Marco bioético en la atención odontológica de pacientes pediátricos en tiempos de COVID-19
}

\author{
Asociación Latinoamericana de Odontopediatría.
} Equipo Interdisciplinario COVID-19

\section{Introducción}

A principios del 2020, un nuevo virus causante del síndrome respiratorio agudo severo (SARS-CoV-2) se diseminó a nivel mundial causando la enfermedad de coronavirus o COVID-19. La tasa de nuevos contagios varía por día y puede ser monitoreada en tiempo real. ${ }^{1}$ Con respecto a la situación actual en Latinoamérica, los casos confirmados de COVID-19 se han incrementado de forma importante. ${ }^{2} \mathrm{La}$ Organización Mundial de la Salud (OMS) ha declarado esta situación como una pandemia debido a los niveles alarmantes de propagación de la enfermedad y en especial por las complicaciones que se pueden suscitar luego del contagio. ${ }^{3}$

Desde el comienzo de la emergencia sanitaria a nivel mundial, los esfuerzos en Latinoamérica para detener la propagación de la enfermedad se han visto afectados negativamente debido al contexto propio del continente, el cual es anterior a la pandemia, e incluye: la pobreza, desigualdad, discriminación, la debilidad estructural de los sistemas públicos de salud y en algunos países, la inestabilidad política e institucional. Los factores antes mencionados han hecho difícil la eficacia de las medidas de confinamiento y distanciamiento social así como otras acciones en concordancia con el Reglamento Sanitario Internacional (RSI) y las recomendaciones emitidas por la OMS y la Organización Panamericana de Salud (OPS).

Como resultado de esta situación, la población de los diferentes países del continente Americano, han sido y continúan siendo, extremadamente afectadas por la pandemia global. Actualmente existe un amplio grupo social con COVID-19, siendo los más afectados aquellos en situación de vulnerabilidad, quienes requieren una atención prioritaria velando por la defensa y protección de sus derechos como seres humanos. Las personas con COVID-19 corren un riesgo especial de transgresión de sus derechos humanos, en particular a la vida y a la salud, por lo que se hace necesaria una adecuada disposición de instalaciones, bienes y servicios sanitarios y médicos, para realizar el diagnóstico y tratamiento. ${ }^{4}$ 
Los odontólogos, higienistas, asistentes dentales y los pacientes siempre han tenido un alto riesgo de infecciones cruzadas debido a su exposición a microorganismos patógenos presentes en la cavidad bucal y en las vías respiratorias. El entorno odontológico implica niveles peligrosamente altos de microorganismos como resultado del contacto cercano con la cavidad bucal del paciente y de la presencia de estos en los aerosoles generados durante algunos tratamientos. ${ }^{5}$

El llamado urgente que han realizado los diferentes sectores de salud de priorizar el enfoque bioético en todos los aspectos relacionados con la atención clínica e incluso la investigación durante la crisis actual de salud pública, ha requerido el trabajo colaborativo de diferentes grupos académicos y clínicos que contribuyan a dar una respuesta bioética a la atención en odontopediatría en este tiempo de pandemia ocasionada por la COVID-19.

\section{La Asociación Latinoamericana de} Odontopediatría (ALOP) ha convocado con éxito a un equipo multidisciplinario para construir un marco bioético que permita una mejor comprensión de la toma de decisiones promoviendo enfoques basados en diferentes niveles de evidencia y sustentados por la ética. Siendo así, este documento ha sido diseñado para emprender un acercamiento al debate relacionado con las preocupaciones éticas que se derivan de la atención odontológica y sus estándares potencialmente críticos durante la contingencia. Dentro de este marco, los odontopediatras podrán reforzar algunos conceptos importantes, entre ellos, los tres deberes principales de la atención médica y odontológica durante una emergencia de salud pública: planificar, salvaguardar y guiar. También se busca brindar detalles que orienten a los diferentes Comités de Ética adscritos a instituciones de salud y académicas para apoyar a los profesionales de la salud que atienden pacientes durante la contingencia.

Los objetivos de este marco bioético son:

1. Destacar la importancia del uso del consentimiento y del asentimiento informado para la realización de tratamientos odontológicos en población pediátrica, así como para la investigación en tiempos de COVID-19.

2. Implementar una guía clara y concisa que provea e incentive al especialista en odontopediatría a la reflexión bioética durante la atención odontológica en tiempos de COVID-19.

\section{Consentimiento y asentimiento informado en odontopediatría en tiempos de COVID-19. Período de cuarentena/ confnamiento - periodo de desescalada}

El consentimiento previo, libre, pleno e informado deriva de derechos reconocidos en el sistema interamericano, como el derecho a la salud, a recibir y a tener acceso a la información, así como a no sufrir injerencias arbitrarias en la vida privada y que, asimismo, es un aspecto central en el desarrollo de la bioética de los derechos humanos, entendido como una herramienta de gran valor para enmarcar y resolver los problemas $y$ dilemas vinculados a la pandemia. ${ }^{4}$

Existe un deber ético dentro del principio universal de autonomía que es el de respetar el derecho del paciente a la autodeterminación y confidencialidad. Este deber ético puede ser particularmente 
desafiante con la pandemia actual, ya que se tiene que actuar dentro del tratamiento aceptable y esto depende de la situación de salud de cada país y región. En el contexto actual, las circunstancias de salud pública obligan al odontopediatra a postergar ciertos tratamientos, en especial tratamientos electivos, hasta que mejore la situación o sea levantado el confinamiento.

Cualquier tratamiento odontológico debe recabar el consentimiento previo, libre e informado del representante legal del paciente. Dicho consentimiento, que en preferencia debe ser obtenido de forma escrita, debe suministrar información oportuna, completa, comprensible, clara, fidedigna y sin tecnicismos, teniendo en cuenta sus particularidades y necesidades específicas. De igual forma se le debe informar al representante legal sobre la evaluación de diagnóstico, tratamiento propuesto, el objetivo del tratamiento, duración probable y los beneficios $\mathrm{y}$ riesgos esperados del tratamiento, los posibles efectos desfavorables de la terapéutica propuesta, las alternativas de tratamiento incluyendo las menos intrusivas y el posible dolor o malestar, los riesgos, beneficios y efectos secundarios de otros tratamientos alternativos. ${ }^{4}$

Para que el representante legal de un paciente pediátrico brinde su consentimiento informado bajo el principio ético de autonomía en tiempos de COVID-19, se debe brindar información clara y precisa sobre los riesgos de la infección viral para el paciente, su familia y la comunidad, al conocer los riesgos, el cuidador puede evaluar con precisión la relación riesgo/ beneficio que se obtendrá del tratamiento odontopediátrico dependiendo de las circunstancias individuales del paciente. Es importante que se diferencien $y$ prioricen aquellos procedimientos que constituyen emergencias o urgencias dentales, de condiciones no emergentes. ${ }^{6}$

Con relación a la confidencialidad de los registros de los pacientes, los pacientes como sus representantes legales deben estar informados sobre la posible necesidad de compartir información identificable en caso de sospecha de contagio dentro del consultorio, de resto el odontólogo y su personal deben continuar manteniendo y respetando la confidencialidad y privacidad de todos los pacientes. ${ }^{6}$

Es importante destacar la importancia de la preservación de la privacidad y la protección de datos personales de las personas con COVID-19, en especial en estos momentos en donde ha habido una proliferación de herramientas digitales y aplicaciones que utilizan los datos personales de la población, e incluyen información personal sensible en el contexto de la pandemia. Existen diferentes normativas y un marco jurídico robusto sobre protección de datos, y el rol que juegan los órganos garantes en el cumplimiento de estos derechos. ${ }^{4}$

En el desarrollo del niño como persona, la madurez, autonomía y plena capacidad de decisión se adquieren mediante un proceso progresivo que dura años. La autonomía permite que la persona decida sobre sus actos individuales y de razón de estos, por lo tanto, la autonomía involucra dos preceptos éticos: todas las personas deben ser tratadas como individuos autónomos y todas las personas con disminución de su autonomía tienen derecho a la protección. Los niños logran plena identificación 
de sus derechos individuales y de los principios éticos universales alrededor de los 14 años, sin embargo, la edad no es la medida óptima de la capacidad mental.

Los niños, adolescentes y otras poblaciones vulnerables pueden tener una capacidad de toma de decisiones disminuida, y en ocasiones no están facultados para dar el consentimiento por si mismos. Los adultos responsables determinarán si la relación riesgo/beneficio es aceptable para permitir la realización de un determinado procedimiento, en especial cuando se trate de procedimientos invasivos, otorgando o no el consentimiento para su realización. Al niño se le debe explicar el procedimiento, de una forma comprensible por él, y solicitar el asentimiento informado.

Entre los 7 y 18 años surge una situación intermedia entre el consentimiento delegado a un adulto y el consentimiento autónomo que se denomina asentimiento. Para lograr este último, se debe suministrar al niño o adolescente información sobre los procedimientos que se le realizarán adaptada a su edad. Privarlo de ella, por una actitud de paternalismo mal entendida, es inmoral porque le anula como persona. Preguntarle al niño y contar con él, es una forma de reconocer su dignidad como persona y su condición como sujeto moral, y esto es así, no solo atendiendo a criterios éticos, sino también legales. El asentimiento es un acto sin valor legal de aceptación que debe ir acompañado siempre de un consentimiento verbal o preferiblemente escrito y firmado por los padres o tutores del menor. Implica un reconocimiento de la autonomía y constituye un acto de gran valor ético. En líneas generales se busca que el consentimiento de los padres no excluya el de los niños. Ante la duda de que no exista mucho acuerdo entre los padres y el menor, el consentimiento no debe ser firmado hasta que no se discuta con el menor y que exprese su voluntad y propiciar el diálogo entre el menor, sus padres y el odontopediatra para llegar a un acuerdo. ${ }^{4,6}$

En este documento se proponen modelos genéricos para el consentimiento informado, así como asentimiento informado, para la realización de tratamiento de odontopediatría durante la pandemia COVID-19. (Anexos 1-3)

\section{Aspectos éticos en la atención clínica de pacientes pediátricos en tiempos de COVID-19}

Los problemas éticos propios de la atención preventiva y curativa en odontopediatría son numerosos y frecuentes. No obstante, en tiempos de epidemias y pandemias como la que se está viviendo en la actualidad por la COVID-19 pueden acarrear una carga significativa para la salud de la población pediátrica y conducir a una desorganización social y económica importante. Aunado a esto, se acentúan algunos dilemas éticos debido al riesgo biológico asociado a la atención odontológica. No obstante, el deber moral indica que se tiene de dar atención oportuna a todo paciente que así lo requiera. En este sentido, es importante tomar conciencia de estos dilemas éticos y de los valores individuales y sociales que constituyen la base del actuar ciudadana, profesional, así como las políticas de salud locales y regionales implementadas.

La Asociación Dental Americana ${ }^{7}$ (ADA) recomienda que se continúen utilizando 
los Principios de Ética y el Código de Conducta Profesional (Código de Ética) como guía en la atención de pacientes durante la pandemia por la COVID-19. Los principios éticos de autonomía, no maleficencia, beneficencia, justicia y veracidad deben mantenerse como pilares fundamentales de la actividad profesional. Las premisas generales del Código de Ética de no hacer daño y poner el bienestar del paciente primero se mantienen. Con frecuencia, las recomendaciones $\mathrm{y}$ anuncios gubernamentales son consistentes con el Código de Ética, sin embargo, pueden requerir que los odontólogos tomen medidas adicionales a las que normalmente se exigen y deben ser cumplidas de acuerdo a las regulaciones nacionales y regionales.

\section{El valor de la vida y de la salud}

Toda vida humana es igualmente valiosa y digna y todos por igual tienen derecho a la vida y a la salud. El odontopediatra debe proveer una atención odontológica de calidad, con todos los recursos que minimicen los riesgos biológicos que de ella se deriven, de forma tal de garantizar el éxito del procedimiento realizado y que los beneficios superen los posibles riesgos que se pudieran originar con la atención.

La atención de pacientes durante la pandemia debe estar orientado en el respeto por la dignidad personal. Se trata de un valor esencial e intrínseco que permite considerar a todo ser humano como un fin en sí mismo, nunca como un medio, un portador de una singularidad $\mathrm{y}$ valor esencial, que lo transforma en un ser único e irrepetible. Entre otras aplicaciones, el respeto por la dignidad impide considerar criterios de asignación de recursos que estén influenciados por cuestiones ajenas a la esencia personal, tales como la edad, la valoración social, la discapacidad, entre otras. Se debe enfatizar la importancia de la calidad y el sentido de la vida para cada uno de los pacientes.

Para ahondar más dentro del valor de la vida y la salud se requiere la aplicación de principios éticos que a continuación se mencionan: ${ }^{8}$

1. Libertad: forma parte de los derechos humanos, que son garantías jurídicas universales que protegen a los individuos o grupos contra acciones que interfieran con sus derechos individuales y contra la dignidad humana. Todas las acciones que se tomen para el control de una epidemia/ pandemia, incluyendo tratamientos médicos y odontológicos, tienen que ejecutarse dentro del más absoluto respeto a los derechos humanos.

2. Justicia: el odontopediatra debe prestar atención a todos por igual, sin discriminaciones de edad, género, raza, condición social y económica. Este principio ético requiere que los riesgos, beneficios y cargas de las acciones en materia de salud, se distribuyan de una manera justa. "Es la distribución justa, equitativa y apropiada en la sociedad determinada por normas justificadas que estructuran los términos de la cooperación social". La salud bucal de la población pediátrica debe ser protegida.

3. Equidad: la distribución de los recursos debe hacerse de acuerdo a las necesidades y urgencias y esto 
debe prevalecer sobre los recursos económicos de los distintos grupos o partes de una sociedad. No obstante, es meritorio destacar que se debe dar prioridad en la atención odontológica a los que tienen mayor gravedad o afecciones bucales o a los más vulnerables. La falta de equidad hace referencia a diferencias en la salud que son innecesarias, evitables y que se consideran injustas o ilegítimas.

4. Solidaridad: representa la responsabilidad y unión compartida entre personas. Los individuos con solidaridad mutua comparten intereses comunes y no están separados por opiniones, propósitos o acciones. La solidaridad está estrechamente relacionada con los principios de justicia y equidad y representa una obligación social que lleva a favorecer la atención de los más vulnerables de una sociedad, más que como una actitud personal. La solidaridad debería ser el principio rector de la salud pública, en general y con mayor énfasis, en el abordaje a la pandemia. La solidaridad se presenta como un dique de contención frente a la desigualdad y la discriminación social. En la atención de la pandemia por la COVID-19, cobra especial significado ya que sus víctimas aumentan en la misma medida que se acrecientan las fragilidades, las inequidades sociales, las vulnerabilidades y el estigma. El verdadero valor de la solidaridad se expresa cuando quien recibe el gesto solidario carece de todo poder, como aquellos que sufren las consecuencias dañinas de la pandemia. Allí se refleja el auténtico sentido humanitario de la fraternidad.
5. Reciprocidad: una relación entre partes se caracteriza por una acción mutua correspondiente.

6. Responsabilidad profesional: éticamente significa hacerse cargo de lo realizado frente al afectado, pero también significa cuidar de otro ser humano vulnerable.

En este sentido, los valores éticos para racionar y distribuir los recursos en tiempos de pandemia convergen en cuatro valores fundamentales:

- Maximizar los beneficios que se puedan producir durante la atención odontológica empleando la menor cantidad de recursos o los que menos riesgo biológico susciten.

- No discriminar. Tratar a todos los pacientes con el mismo respeto $y$ dedicación.

- Promover y recompensar el trabajo del personal de salud durante la pandemia.

- Dar prioridad a las situaciones más graves y a los más enfermos. ${ }^{8}$

\section{Importancia de los valores éticos en tiempos de pandemia}

Durante situaciones de emergencia de salud pública, los profesionales de la salud y en este caso, los odontopediatras, deben equilibrar varios deberes: la atención centrada en el paciente, la ética profesional y la promoción de la igualdad y equidad en la distribución de riesgos y beneficios en la sociedad. ${ }^{9}$

La gran mayoría de los clínicos han recibido capacitación dirigida a una práctica profesional centrada en el 
paciente y tan sólo un pequeño porcentaje la ha recibido para la atención guiada por consideraciones de salud pública. ${ }^{10} \mathrm{La}$ pandemia cambió el contexto y la forma de distribuir los recursos humanos y económicos y esto ha generado tensión especialmente en donde se han agravado las barreras de acceso a la atención médica y la disponibilidad de los recursos. ${ }^{11,12}$

Tradicionalmente la atención clínica se centra en el paciente, por lo que se basa en las preferencias y valores de cada paciente. Por el contrario, cuando se trabaja en el contexto de una emergencia de salud pública como la actual, se interrumpe la atención normal y se promueve la salud de la población minimizando la morbilidad y la mortalidad mediante el uso prudente de los recursos y de las estrategias. ${ }^{13,14}$

El ejercicio profesional debe tener como objetivo principal actuar éticamente, brindando un alto nivel de atención, limitando la incertidumbre o la angustia sobre cómo proceder, sin importar si se desarrolla en circunstancias normales o de emergencia. ${ }^{9}$ En el contexto de la pandemia, se deben establecer protocolos que permitan priorizar a los pacientes en función de la necesidad de tratamiento que requieran y en función de las posibles limitaciones de los recursos. . $^{11,16}$

Los deberes éticos que responden a la pandemia por la COVID-19 y que deben equilibrarse son: el deber de la atención médica que requiere fidelidad al paciente, alivio del sufrimiento y respeto a los derechos y preferencias de los pacientes, el deber del cuidado y sus ramificaciones como enfoque principal de la ética clínica y el deber de promover la equidad para el cuidado de las personas asegurando una distribución de beneficios y riesgos adecuada en la sociedad. Esto genera divisiones importantes como la promoción de la seguridad pública, la protección de la salud comunitaria y la asignación de recursos limitados, entre otras. Tanto los deberes mencionados como sus ramificaciones son el foco principal de la ética de la salud pública. ${ }^{11,17}$

Es deber del profesional de la salud el saber planificar y gestionar la incertidumbre que se pueda derivar del manejo de desafíos éticos previsibles y emergentes durante la pandemia sobre cómo "hacer lo correcto" en la práctica clínica cuando los deberes y valores entran en conflicto. Estos desafíos suelen afectar la fortaleza del personal de salud y les hacen confrontarse a sí mismos. La planificación de los desafíos éticos en una crisis de salud pública como la actual incluye la identificación de posibles decisiones de triage, herramientas y procesos, escasez de personal, cambios en los espacios y las dificultades relacionadas con la disminución de los suministros. ${ }^{17}$

En casos de emergencia de salud pública el llamado es a dar respuesta a la mayor demanda de atención con la menor cantidad de recursos. Es posible que deban modificarse conductas terapéuticas habituales, adaptando la atención según los protocolos de control de infecciones, el uso racional de recursos, yla disponibilidad de personal, espacio y suministros. Sin embargo, las consideraciones bioéticas destacan que debe beneficiarse al paciente crítico que espera atención odontológica especializada. ${ }^{11,12}$

Durante la crisis de salud pública de COVID-19, es necesario salvaguardar a todas las personas vulnerables incluyendo a los trabajadores dela salud y a las personas susceptibles a mayores complicaciones 
derivadas de la enfermedad debido a factores como la edad, condiciones de salud subyacentes y aquellos con barreras preexistentes para el acceso a la atención médica. ${ }^{11}$ En el caso de la odontología, se debe reconocer que todo el personal como población vulnerable y de alto riesgo.

El acompañamiento y seguimiento de los pacientes, en particular de los más vulnerables, cobra especial importancia durante la pandemia, cumpliendo con la responsabilidad profesional. El uso de herramienta de atención a distancia, como la teleodontología, permita al clínico mantener contacto con el paciente, realizar actividades de promoción de salud, mostrar solidaridad, además de orientar y permitir la realización del mejor triage posible, para determinar los casos que deben ser atendidos de forma presencial. ${ }^{18}$

Los Comités de Ética Institucionales pueden apoyar a los profesionales de la salud, pacientes y a los miembros de la familia en la toma de decisiones informadas, basadas tanto en los derechos de los pacientes como en la responsabilidad profesional en materia de salud, considerando los cuatro principios de la bioética: autonomía, justicia, beneficencia y no maleficencia. ${ }^{15}$ Igualmente, deben prepararse para el servicio durante emergencias de salud pública reconociendo la importancia de promover la igualdad de las personas y la equidad en la distribución de los beneficios y riesgos para la sociedad, apoyando de la mejor manera la práctica clínica durante la pandemia, centrado en la atención al paciente, la gestión con personal reducido y el desarrollo de estrategias para abordar la angustia moral del personal bajo condiciones de crisis. ${ }^{15,19-21}$

\section{Marco normativo}

Las finalidades de toda atención o servicio de salud son la protección de la vida y de la salud tanto física como mental, la optimización del bienestar de forma integral, el no abandono, el respeto de la dignidad como ser humano y su autodeterminación, haciendo uso del máximo de los recursos disponibles para un mejor tratamiento en la medida de las posibilidades.

Para la protección de la población, los Estados deben guiar las medidas que se adopten bajo los principios de igualdad y no discriminación de conformidad con los estándares interamericanos e internacionales de derechos humanos. En este sentido, resultan de relevancia la Declaración sobre Bioética y Derechos Humanos de la Unesco, así como los aspectos fundamentales de la ética de la salud pública reconocida por las instituciones especializadas nacionales e internacionales en la materia. Las orientaciones técnicas de la OMS y de la OPS sobre el virus, así como los pronunciamientos de los Comités Nacionales de Bioética, sociedades científicas y médicas y otras instancias médicas.

Cada profesional es responsable de conocer y acatar las leyes, regulaciones y normativas que rigen los aspectos éticos de la atención odontológica de su localidad.

Para la protección del derecho a la salud de la población pediátrica, es necesario reconocer y garantizar el derecho que tienen de recibir una prestación adecuada de los servicios de salud, estos deben estar inmersos en un marco normativo y en protocolos de atención y tratamiento con 
parámetros claros de atención. Asimismo, estos deben ser constantemente revisados y actualizados, según la mejor evidencia científica. ${ }^{4}$

\section{La Federación Dental Internacional} $(\mathrm{FDI})^{22}$ publicó unas recomendaciones importantes a considerar para la práctica odontológica y la promoción de la salud bucodental durante la pandemia del coronavirus. En este marco, la FDI estableció unos principios fundamentales:

- La salud bucal es un servicio público esencial, fundamental para el bienestar y la salud en general y debe ser accesible para toda la población, por lo tanto, las autoridades correspondientes deben garantizar su acceso.

- Los profesionales de la odontología deben estar presentes en los debates y toma de decisiones relacionadas con la regulación y orientación de la atención médica durante la pandemia.

- Las autoridades deben facilitar el apoyo financiero y administrativo necesario a los odontólogos para reducir la carga financiera sobre las prácticas dentales y garantizar la continuación de una atención adecuada durante y después de la COVID-19.

- Toda la normativa y las orientaciones dirigidas hacia los profesionales de la odontología deben tener en cuenta la situación y las necesidades de salud pública de cada país y no solo el riesgo de infección individual de los profesionales y de los pacientes.

- Los odontólogos deben seguir estrictamente todas las pautas y regulaciones nacionales vigentes, incluidas las relacionadas con el equipo de protección personal (EPPs), los procedimientos de los diferentes tratamientos y los protocolos de admisión del paciente. Las autoridades deben garantizar el acceso a los EPPs a precios asequibles.

- Los profesionales de la odontología tienen la responsabilidad de garantizar la atención en caso de emergencia, siempre que sea posible.

- Es un deber utilizar todas las oportunidades para reforzar con mensajes de prevención de enfermedades en cavidad bucal con el objetivo de reducir la necesidad de tratamientos, las consultas electivas y los costos por atención médica.

- Los odontólogos tienen la responsabilidad de proteger la salud y el bienestar de su personal y de los pacientes.

- Las investigaciones sobre la COVID-19 y su transmisión, incluidas las consideraciones específicas para la práctica dental son necesarias para permitir una orientación adecuada al respecto.

\section{Sobre el tratamiento odontológico en niños durante la pandemia COVID-19}

El periodo de incubación de este virus varía entre individuos y puede ser de 2 y 14 días. Adicionalmente, los niños pueden estar asintomáticos o presentar síntomas leves e inespecíficos de la enfermedad, por lo que, tanto ellos como sus padres y representantes deben considerarse portadores potenciales del virus a menos que se demuestre lo contrario. La transmisión del virus es conocida y se ha ampliado en publicaciones anteriores 
pertenecientes al Centro de Recursos COVID-19, sin embargo, siempre es importante destacar que el odontopediatra como personal de salud se expone a situaciones cuyo riesgo de contagio es potencialmente alto. ${ }^{23}$

Bioéticamente, propiciar la realización de tratamientos odontológicos en población pediátrica cuando esta la demande, constituye un deber ético y es responsabilidad del odontólogo prestar una atención de alta calidad, pero que a su vez represente el menor riesgo biológico colectivo.

Los tratamientos deben ser realizados considerando como prioridad la vida y salud general de los pacientes incluyendo su salud bucal, por lo que el tratamiento de urgencias en odontopediatría siempre estará justificado. No obstante, es deber del odontólogo tratante y parte de su responsabilidad como profesional de la salud en este momento histórico, el velar por el cumplimiento de los protocolos internacionales de bioseguridad dentro del consultorio.

Otro aspecto a resaltar es la importancia de una práctica eficiente simplificando los procedimientos para disminuir la generación de aerosoles y de esta forma disminuir el riesgo de contagio por infección cruzada, respetando y honrando el principio de beneficencia y no maleficencia. Actualmente existen opciones de tratamiento que minimizan o eliminan los procedimientos generadores de aerosoles, muchos de los cuales se basan en la cariología contemporánea, están bien documentados en la literatura científica y son mínimamente invasivos por su naturaleza. ${ }^{24}$

La orientación que se ofrece nunca puede cubrir todas las circunstancias posibles $y$ se debe ejercer con responsabilidad profesional y social a la hora de tomar decisiones sobre si se debe o no brindar un determinado tratamiento. Es importante que el odontopediatra siga las pautas locales, nacionales y de la región. Durante la pandemia de COVID-19 es importante extremar el control de infecciones en defensa de la salud de todos. Una vez que se empiecen a levantar las restricciones de la práctica odontológica se recomienda que se mantenga la realización de procedimientos que generen la menor cantidad de aerosoles y sean mínimamente invasivos. Entre estos se destaca el tratamiento restaurador atraumático (ART), el sellado de lesiones cariosas con sellante de fosas y fisuras, el empleo de fluoruro diamino de plata, la remoción selectiva de caries y la técnica Hall. ${ }^{24}$

Hay que tomar en cuenta que algunos Estados progresivamente están adoptando transiciones hacia la reactivación plena de las actividades, resultando necesario que se adopten medidas necesarias para minimizar los riesgos de contagio, prevenir rebrotes masivos de la COVID-19. Esto incluye políticas públicas dirigidas a asegurar la disponibilidad de pruebas diagnóstico, mejorar la accesibilidad a los servicios de salud necesarios para asegurar, que de haber un aumento de los casos, se disponga de los recursos médicos, infraestructura hospitalaria y la disponibilidad de insumos. ${ }^{4}$

\section{Consideraciones en bioseguridad}

Dado que la transmisión de la infección se da por las gotículas suspendidas en el aire, siendo esta la principal vía de propagación del virus, se recomienda 
que el equipo de trabajo utilice todas las medidas de barreras recomendadas por entes internacionales, a saber: gafas y máscara protectora, máscara facial N95 o similar, guantes, gorro y ropa de protección adecuada para toda la atención odontológica que se preste durante el periodo epidémico. ${ }^{25}$

\section{Investigación en niños y adolescentes en tiempos de pandemia.}

De acuerdo con las normas éticas internacionales para la investigación en el área de salud en seres humanos, ${ }^{25-27}$ la participación de niños y adolescentes resulta indispensable para la investigación de enfermedades o de condiciones patológicas a la que son particularmente susceptibles este grupo etario y los estudios deben estar canalizados en el trabajo "con" niños y no "en" niños, es decir, basados en el concepto moderno de la investigación centrada en el niño, considerando su opinión y decisión de participar en el proceso de investigación.

En este sentido, la participación del niño debe ser activa durante toda la investigación, su opinión debe ser tomada seriamente en cuenta y dependiendo de la edad, pueden ser competentes y reflexivos al informar sus propias experiencias.

La investigación en odontología es esencial para proporcionar el mejor tratamiento clínico al niño y adolescente el cual debe estar basado en evidencia científica sólida y disponible sobre la eficacia terapéutica de los diferentes procedimientos, así como de los diversos materiales dentales que serán utilizados y la seguridad de los mismos en esta población. ${ }^{6}$
Para que la investigación pueda considerarse científica, se debe basar en una serie de valores que surgen del mismo carácter de la ciencia, cuyo fin es la búsqueda de la verdad objetiva. Es importante hablar de una ética de la investigación, es decir, de una investigación con conciencia, cuyos resultados sean correctamente utilizados, en especial cuando la población a estudiar es pediátrica. ${ }^{28}$

Es responsabilidad del investigador garantizar que su estudio sea de calidad, tanto en lo que se refiere a la originalidad, relevancia y valor para su área de conocimiento, como en destacar la importancia de considerar los aspectos éticos concernientes a la investigación. La honestidad debe caracterizar toda investigación (desde el planteamiento inicial, el diseño del estudio, hasta la difusión y publicación de los resultados) y para que esto sea posible, el investigador o grupo de investigadores deben ser honestos.

Un comité de ética no suple la moralidad del investigador, que queda reflejada en múltiples aspectos y momentos del proceso: la recolección de los datos de manera cuidadosa y metódica, el registro meticuloso del trabajo realizado, la interpretación realista de los resultados, sin forzar o ajustar los datos a las hipótesis que interesan al investigador, etc.

Lo primero a tener en cuenta en la investigación odontológica en niños y adolescentes, es que la verdad científica está al servicio de la persona y no al contrario. Por lo tanto, la verdad en medicina no es un fin en sí mismo, sino un medio al servicio del hombre, el cual 
sí se considera un fin per se. Esto no ha de significar un obstáculo ni un elemento disuasorio para el avance del conocimiento a través del método científico, sino que, más bien, asegura una investigación de calidad y bien elaborada, acorde con su finalidad última de mejorar la calidad de vida del ser humano en el respeto a su dignidad. ${ }^{29}$

También debe tenerse en cuenta la protección de la dignidad del ser humano que es objeto de investigación, contando con su consentimiento libre y bien informado acerca de los riesgos y posibles consecuencias de los procedimientos realizados sobre su persona. ${ }^{29}$

La ética en investigación enfatiza la responsabilidad en el ejercicio de la actividad científica, que incluye evitar sufrimientos innecesarios a los sujetos que participan en la investigación. Esta responsabilidad también incluye el fomentar de manera especial la reflexión ética que permita desarrollar el increíble potencial de la investigación científica, en aras de mejorar las condiciones de vida en el futuro y no de deteriorarlas. ${ }^{29}$

Por ultimo se debe garantizar la total transparencia en la investigación. Es responsabilidad del investigador ser siempre capaz de dar razón de su trabajo, respetando asî, por un lado, la opinión de la comunidad científica al evaluar sus descubrimientos $\mathrm{y}$, por otro lado, siendo consciente del impacto social y la responsabilidad hacia la sociedad que tiene la actividad científica. ${ }^{29}$

Debido a la excepcional crisis sanitaria de la que se está siendo testigo en la actualidad debido a la propagación de la COVID-19, impone el deber ético de hacer investigación a fin de mejorar la prevención y la atención sanitaria de la población mundial. Esta investigación, es esencial para comprender la enfermedad, sus mecanismos de contagio y expansión, a fin de formular intervenciones y prácticas que sean eficientes $y$ eficaces y que permitan contener el contagio. De igual forma, la investigación en esta materia permite también evaluar la seguridad y validez de los exámenes diagnósticos, tratamientos, vacunas y estrategias de manejo. En líneas generales, la situación no justifica la inobservancia de las pautas establecidas, guías y recomendaciones de los organismos internacionales vigentes, las cuales deben respetarse en todos los casos. Por ello, es imprescindible que se promueva la investigación sin quitar rigurosidad a la evaluación ética y metodológica de los estudios para cumplir el fin último que es la protección de los sujetos de investigación. En este orden de ideas cabe destacar la importancia de la obtención del consentimiento informado que permita asegurar que se respete la autonomía de todos los participantes y que su decisión es voluntaria.

A este documento anexamos modelos base de consentimiento y asentimiento informado para la realización de investigación científica en odontopediatría durante COVID-19. (Anexos 4 - 6)

La situación particular relacionada a la virulencia y la facilidad de contagio del COVID-19, encierra desafíos vinculados a la documentación del proceso de consentimiento informado. En ese orden, cuando los mecanismos habituales no son posibles, es necesario considerar formas alternativas (como por ejemplo contactar a los participantes por videollamada, 
desarrollar un registro de consentimientos informados por medios audiovisuales o imágenes digitales, obtención de consentimientos orales complementados con confirmación por correo electrónico y asiento en la Historia Clínica con la firma de uno o varios profesionales de la salud). La pertinencia y aceptación de las variantes dependerá de la normativa interna de la institución en la que se realice la investigación, de las regulaciones locales y con la autorización del Comité de Ética Institucional. ${ }^{30}$

\section{Recomendaciones}

La pandemia por la COVID-19 plantea cuestiones éticas y legales, en las que se incluyen prioritariamente la asignación de recursos y el acceso a la atención médica. Los cuatro principios éticos universales: autonomía, beneficencia, no maleficencia y justicia, pueden ayudar a los odontopediatras en la toma de decisiones y en la reflexión moral que se deriva de la atención odontológica durante la pandemia.

El odontopediatra tiene el deber de actuar priorizando el principio de beneficencia, pero respetando su dignidad y autonomía. Durante situaciones como la actual, la dignidad humana es el más alto de todos los valores y es independiente de la posición social o de la utilidad para los demás. En tal sentido, los tratamientos deben proporcionarse independientemente de la edad, género, origen o etnia, color, lugar de residencia, estatus social o económico (principio de justicia).

La COVID-19 amplifica los problemas de acceso a la atención médica y los problemas relacionados con la escasez de recursos. Por lo tanto, está éticamente justificado el reprogramar o cancelar procedimientos electivos, sin embargo, el deber ético es el de proporcionar una atención esencial y adecuada, tratando a los pacientes de acuerdo a sus necesidades, empleando la menor cantidad de recursos disponibles y disminuyendo en lo posible la generación de aerosoles que pudieran aumentar el riesgo de contagio y la propagación de la enfermedad. ${ }^{31}$

\section{Autores}

Desarrollo de este artículo liderado por: Rosa Gabriela Rondón (Venezuela), Gabriel Zambrano (Venezuela), Martha Mutis (Estados Unidos de América), Paola Beltri (España), Olga Cortés Lillo (España), como parte del Equipo Interdisciplinario COVID-19 de la Asociación Latinoamericana de Odontopediatría.

Equipo Interdisciplinario COVID-19 de la Asociación Latinoamericana de Odontopediatría: Jenny Abanto (Brasil), Mariana Armada (Argentina), Paola Beltri (España), Marisol Carrillo Tabakman (Paraguay), Haydée Casaretto (Argentina), Jorge Luis Castillo (Perú), Mónica Gladys Cesetti (Argentina), Bertha Angélica Chávez González (Perú), Ana Claudia Rodrigues Chibinski (Brasil), Salomon Alberto Cohen (Argentina), Olga Cortés Lillo (España), Luzia Ana Da Silva de Carballo (Venezuela), Gonzalo De la Fuente Alvarez (Chile), Renée Di Nallo (Argentina), María Débora Elizabeth Dricas (Argentina), Sandra Echevarria (Brasil), Piedad Cecilia Echeverry Marin (Colombia), Laura Fedelli (Argentina), Carlos Flores-MIr (Canadá), Andrea Virgina González Carfora (Chile), Lina María Hernández Salas (Colombia), Francisco José Hernández Restrepo (Colombia), Maria Teresa Ibañez Rodriguez (Bolivia), José Carlos Pettorossi Imparato (Brasil), Alejandra Lipari Valdés (Chile), Daniela Madrigal López (Costa Rica), Daniela Catalina Martínez Camus (Chile), María Gabriela Martínez Vásquez (Venezuela), A. Carolina Medina Díaz (Venezuela), Kelly Maria Silva Moreira (Brasil), Elías M. Morón (Estados Unidos de América), Martha Mutis (Estados Unidos de América), Camila Palma (Perú), Gladys Mabel Peña (Argentina), Adriana Pistochini (Argentina), Paloma Planells 
(España), Gabriel Politano (Brasil), Matias RiosErazo (Chile), Adriana Maria Rubiano Pinzon (Colombia), Karla Mayra Rezende (Brasil), Rosa Gabriela Rondón (Venezuela), Gabriela Scagnet (Argentina), Rosemary Sogbe de Agell (España), Marina Tavares Costa Nóbrega (Canadá), Jorgelina Valente (Argentina), Ernesto Venegas De Herrera
(República Dominicana), Rosa Helena Wanderley Lacerda (Brasil), Ana Clara Zabala (Argentina), Gabriel Zambrano (Venezuela).

Información completa del Grupo Interdisciplinario COVID-19 de la Asociación Latinoamericana de Odontopediatría

\section{Referencias bibliográficas}

1. Mallineni SK, Innes NP, Raggio DP, Araujo MP, Robertson MD, Jayaraman, J. Coronavirus disease (COVID-19): Characteristics in children and considerations for dentists providing their care. Int J Paediatr Dent. [Internet]: 2020 [Consultado: 2 Junio 2020]; 30: 245-50. Disponible en: https://doi.org/10.1111/ ipd.12653

2. BBC News Mundo. Coronavirus en América Latina: los países de la región donde más está creciendo el número de contagios de covid-19 [Internet]: BBC; Mayo 2020 [Consultado: 2 Junio 2020]. Disponible en: https://www.bbc.com/mundo/noticias-america-latina-52667117

3. Organización Mundial de la Salud. Brote de enfermedad por coronavirus (COVID-19). [Internet]: Organización Mundial de la Salud; 2020 [Consultado: 2 Junio 2020]. Disponible en: https://www.who.int/es/ emergencies/diseases/novel-coronavirus-2019?gclid=Cj0KCQjw9b_4BRCMARIsADMUIyr1ljG9EJJo_sEtfj7TG_qGskQacPg1DxvxrA6_hr6LHJJz43mpAMgaAtU-EALw_wcB

4. Comisión Interamericana de Derechos Humanos. Derechos humanos de las personas con Covid-19 [Internet]: Organización de Estados Americanos; Abril 2020 [Consultado: 20 Julio 2020]. Disponible en: http://www.oas.org/es/cidh/decisiones/pdf/Resolucion-4-20-es.pdf

5. Checchi V, Bellini P, Bencivenni D, Consolo U. COVID-19 dentistry-related aspects: a literature overview. Int Dent J [Internet] 2020 [Consultado: 2 Junio 2020]; doi:10.1111/idj.12601. Disponible en: https://onlinelibrary.wiley.com/doi/full/10.1111/idj.12601

6. Garrocho-Rangel A, Cerda-Cristerna B, Pozoz-Guillen A. Bioethical Issues in Conducting Pediatric Dentistry Clinical Research.J Clin Pediatr Dent [Internet] 2018; [Consultado: 2 Junio 2020]; 42(2):85-90. Disponible en: https://jocpd.org/doi/10.17796/1053-4628-42.2.1

7. American Dental Association. Guidance on continuing to practice ethically during COVID-19. [Internet] 2020 [Consultado: 25 Junio 2020]; Disponible en: https://success.ada.org/ /media/CPS/Files/COVID/ ADA_COVID_How_Do_I_Ethically_See_Patients_During_Pandemic.pdF

8. Poleo J. Ética médica y Covid-19 Comisión Ética Sociedad de Médicos. Asociación de Bioética Clínica. Caracas. 2020: 1-37.

9. Institute of Medicine. Crisis Standards of Care: Systems Framework for Catastrophic Disaster Response. [Internet] Washington, DC; 2012 [Consultado: 25 Junio 2020]; Disponible en: http://www.nationalacademies.org/hmd/ /media/Files/Report\%20Files/2012/Crisis-Standards-of-Care/CSC_rb.pdf.

10. Institute of Medicine. Engaging the Public in Critical Disaster Planning and Decision Making. Workshop summary. [Internet] Washington, DC; 2013 [Consultado: 25 Junio 2020]; Disponible en: http://www. nationalacademies.org/HMD/Reports/2013/Engaging-the-Public-in-Critical-Disaster-Planning-and-Decision-Making.aspx.

11. The Hastings Center. Ethical Framework for Health Care Institutions Responding to Novel Coronavirus SARS-CoV-2 (COVID-19): Guidelines for Institutional Ethics Services Responding to COVID-19. March $16,2020$.

12. Minnesota Department of Health. Minnesota Crisis Standards of Care Framework: Health Care Facility Surge Operations and Crisis Care [Internet] Minnesota; 2020. [Consultado: 25 Junio 2020]; Disponible en: https://www.health.state.mn.us/communities/ep/surge/crisis/framework_healthcare.pdf.

13. Christian MD, Hawryluck L, Wax RS, Cook T, Lazar NM, Herridge MS, Muller MP, Gowans DR, Fortier W, Burkle FM. Development of a triage protocol for critical care during an influenza pandemic. CMAJ. [Internet] 2006; [Consultado: 2 Junio 2020]; 175(11): 1377-81. Disponible en: https://www.ncbi.nlm.nih. gov/pmc/articles/PMC1635763/\#_ffn_sectitle 
14. Hick JL, Hanfling D, Wynia MK, Pavia AT. Duty to Plan: Health Care, Crisis Standards of Care, and Novel Coronavirus SARS-CoV-2 [Internet] Washington, DC. NAM PERSPECTIVES. Discussion paper. National Academy of Medicine; 2020 [Consultado: 2 Junio 2020]. Disponible en: https://doi.org/10.31478/202003b

15. Italian Society for Anesthesia, Analgesia, Resuscitation, and Intensive Care. Clinical Ethics Recommendations for Admission to Intensive Care and for Withdrawing Treatment in Exceptional Conditions of Imbalance between Needs and Available Resources [Internet]. Italia: Italian Society for Anesthesia, Analgesia, Resuscitation, and Intensive Care. 2020 [Consultado: 2 Junio 2020]. Disponible en: https://www. academia.edu/42213831/English_translation_of_the_Italian_SIAARTI_COVID-19_Clinical_Ethics_Recommendations_for_Resource_Allocation_3_6_20

16. Whelan A, Young G, Catanese VM. Medical Students and Patients with COVID-19: Education and Safety Considerations. American Association of Medical Colleges [Internet]. 2020 [Consultado: 2 Junio 2020]. Disponible en: https://www.aamc.org/system/files/2020-03/Role\%20of\%20medical\%20students\%20 and\%20COVID-19-FINAL.pdf.

17. Adams JG, Walls RM. Supporting the Health Care Workforce during the COVID-19 Global Epidemic. JAMA [Internet]. 2020. [Consultado: 2 Junio 2020]; 323(15):1439-40. Disponible en: https://jamanetwork. com/journals/jama/fullarticle/2763136

18. Equipo de Trabajo Multidisciplinario de la Revista de Odontopediatría Latinoamericana. Teleodontología: Aplicación a la Odontopediatría durante la pandemia COVID-19. Rev Odontoped Latinoam. 2020; 10, (2), Obtenible en: https://www.revistaodontopediatria.org/ediciones/2020/2/art-3/

19. Institute of Medicine. Crisis Standards of Care: A Toolkit for Indicators and Triggers. Report brief. [Internet] Washington, DC; 2013 [Consultado: 25 Junio 2020]; Disponible en: http://www.nationalacademies. org/hmd/ /media/Files/Report\%20Files/2013/CSC-Triggers/CSC-Triggers-RB.pdf

20. Altevogt BM, Stroud C, Hanson SL, Hanfling D, Gostin LO. Guidance for establishing crisis standards of care for use in disaster situations [Internet] Institute of Medicine of the National Academies; 2009. [Consultado: 25 Junio 2020]; Disponible en: http://www.inovaideas.org/emergency_books/2.

21. New York State Department of Health, New York State Task Force on Life and the Law. Ventilator Allocation Guidelines. [Internet]. New York; 2015. [Consultado: Julio 2020] Disponible en: https://www.health. ny.gov/regulations/task_force/reports_publications/docs/ventilator_guidelines.pdf.

22. Consejo General de Colegios de Dentistas de España. La FDI elabora un decálogo sobre Odontología y salud oral durante la pandemia por la Covid-19 [Internet]. España: Consejo Dentistas; Junio 2020 [Consultado: Julio 2020]. Disponible en: https://www.consejodentistas.es/comunicacion/actualidad-consejo/ notas-de-prensa-consejo/item/1815-la-fdi-elabora-un-decalogo-sobre-odontologia-y-salud-oral-durantela-pandemia-por-la-covid-19.html

23. Peng X, Xu X, Li Y, Cheng L, Zhou X, Ren B. Transmission routes of 2019-nCoV and controls in dental practice. Int J Oral Sci [Internet] 2020 [Consultado: 2 Junio 2020]; 12(9). Disponible en: https://doi. org/10.1038/s41368-020-0075-9

24. Asociación Latinoamericana de Odontopediatría. Grupo de Trabajo Interdisciplinaro COVID-19. Tratamiento de la enfermedad de caries en época de COVID-19: protocolos clínicos para el control de aerosoles. Rev Latinoamericana Odontoped [Internet] 2020 [Consultado: 2 Junio 2020]; 10(2). Disponible en: https://www.revistaodontopediatria.org/ediciones/2020/2/art-2/

25. Asociación Latinoamericana de Odontopediatría. Grupo de Trabajo Interdisciplinaro COVID-19. Ruta de atención para procedimientos de Odontología Pediátrica durante la etapa de confinamiento o cuarentena de la pandemia COVID-19. Revista de Odontopediatría Latinoamericana. 2020; 10(2): https://www. revistaodontopediatria.org/ediciones/2020/2/art-1/.

26. van Delden J, van der Graaf R. Revised CIOMS International Ethical Guidelines for Health-realted Research Involving Humans.J Am Med Assoc [Internet] 2016 [Consultado: 2 Junio 2020]; 317(2): 1-119. Disponible en: https://cioms.ch/wp-content/uploads/2017/01/WEB-CIOMS-EthicalGuidelines.pdf

27. World Medical Association Declaration of Helsinki. Ethical principles for medical research involving human subjects. J Am Med Assoc. [Internet] 2013 [Consultado: 2 Junio 2020]; 310(20):2191-4. Disponible en: https://pubmed.ncbi.nlm.nih.gov/24141714/

28. Galan M. Etica de la investigación. Revista Iberoamericana de Educación. 2010;54(4):1-2.

29. Martin S. Aplicación de los principios éticos a la metodología de la investigación. Enferm Cardiol. [Internet] 2020 [Consultado: 25 Junio 2020]; 2013;1(1-2):27-30. Disponible en: https://www.researchgate.net/ publication/340495940_Guidelines_for_dental_care_provision_during_the_COVID-19_pandemic 
30. Ministerio de salud GCABA. Recomendaciones del CCE referidas al Consentimiento Informado en investigaciones sobre COVID 19 [Internet] 2020 [Consultado: 25 Junio 2020]; 10(2). Disponible en: https:// www.buenosaires.gob.ar/sites/gcaba/files/recomendaciones_del_cce_referidas_al_consentimiento_informado_en_investigaciones_sobre_covid_19.pdf

31. Gurzawska-Comis K, Becker K, Brunello G, Gurzawska A, Schwarz F. Recommendations for Dental Care during COVID-19. J Clin Med [Internet] 2020 [Consultado: 2 Junio 2020]; 9(1833): 1-15. Disponible en: https://encyclopedia.pub/1722

Recibido: $15 / 06 / 2020$

Aceptado: 30/07/2020

Correspondencia: e-mail: editor@revistaodontopediatria.org 


\section{Anexo 1}

Cod. 608-190

\section{Consentimiento Informado para Tratamiento de Odontopediatría en tiempos de COVID-19.}

YO, con el documento de identidad:

tutor legal del paciente: manifiesto que se me ha proporcionado información oportuna, completa, comprensible, clara, precisa, sin tecnicismos y fidedigna en respeto de mis derechos y de los de mi representado y como expresión de valor de mi autonomía y libertad de decisión y en tal sentido consiento que mi representado reciba tratamiento odontológico durante la pandemia por la Covid-19. Por lo tanto, entiendo:

- Que el periodo de incubación de este virus es largo y durante el cual sus portadores incluyendo los pacientes pediátricos pueden estar asintomáticos o presentar síntomas leves e inespecíficos de la enfermedad, lo que lo hace altamente contagioso.

- Que debido a la limitación para la realización de pruebas virales y el tiempo de espera para su resultado, es difícil determinar quién es portador del virus y quién no al momento de la consulta.

- Que los procedimientos odontológicos pueden generar aerosoles que permiten la diseminación de la enfermedad. La naturaleza ultrafina del aerosol que producen los equipos odontológicos le permite permanecer suspendido en el aire por minutos o hasta horas, pudiéndose transmitir el virus.

Afirmo que:

- He suministrado información veraz sobre mi estado de salud y el de mi representado y he respondido con sinceridad las preguntas en relación con este aspecto que me ha formulado el especialista del consultorio (iniciales).

- Confirmo que ni mi representado ni yo hemos presentado, en los últimos 14 días, ninguno de los síntomas de la siguiente lista: fiebre, dificultad respiratoria, tos seca, secreción nasal, dolor de garganta, ni hemos estado en contacto con personas con confirmación de COVID-19 o con cuadro respiratorio agudo en las últimas dos semanas (iniciales)

- Se me ha dado información pertinente sobre los protocolos de bioseguridad implementados en el consultorio dental para minimizar el riesgo de contagio y ofrecer un entorno lo más seguro posible.

- Comprendo que a pesar de todos los cuidados, la eventual emisión de aerosoles y la proximidad entre el paciente y el equipo odontológico, así como la realización de procedimientos en la cavidad bucal, hacen que el riesgo cero no se pueda garantizar (iniciales)

- Comprendo que se me ha suministrado información completa sobre el diagnóstico, plan de tratamiento, objetivos y alternativas de tratamiento y que debido a la contingencia actual las opciones terapéuticas minimizan o eliminan procedimientos generadores de aerosoles, siendo mínimamente invasivas para el paciente y están sustentadas en la literatura científica.

(iniciales)

- Luego de suministrada toda la información, de que se me aclararan satisfactoriamente las preguntas que realicé y de que se me permitiera analizar sin presión, ni coacción los riesgos y beneficios del tratamiento odontológico que requiere mi representado, en pleno uso de mis facultades y haciendo valor a mi autonomía, decido consentir la realización del mismo o rechazarlo

Nombre: Odontólogo Tratante:
Firma:

Firma:

Lugar:
DNI O CC:

DNI O CC:

Fecha:

\section{ALQP ASOCIACIÓN LATINOAMERICANA DE ODONTOPEDIATRÍA}

Vol 10 N$^{\circ} 2$ Julio-Diciembre $2 0 2 0 \longdiv { \text { e- } 6 2 0 8 1 6 }$ 


\section{Anexo 2}

Cod. 608-191

\section{Asentimiento Informado para Tratamiento de Odontopediatría en tiempos de COVID-19.}

Hola! Soy tu odontopediatra y me dedico a cuidar la salud de toda tu boca. Se que en los últimos meses las cosas han cambiado mucho y ahora debemos protegernos los unos a los otros usando tapabocas (mascarilla), lavando nuestras manos constantemente con agua y jabón y en mi caso, debo usar un uniforme nuevo que me ayuda a resguardarme a mi y a ti mientras hago mi trabajo, eso es porque existe un virus nuevo llamado coronavirus que produce una enfermedad llamada Covid-19 que nos puede enfermar y debemos evitar que eso ocurra.
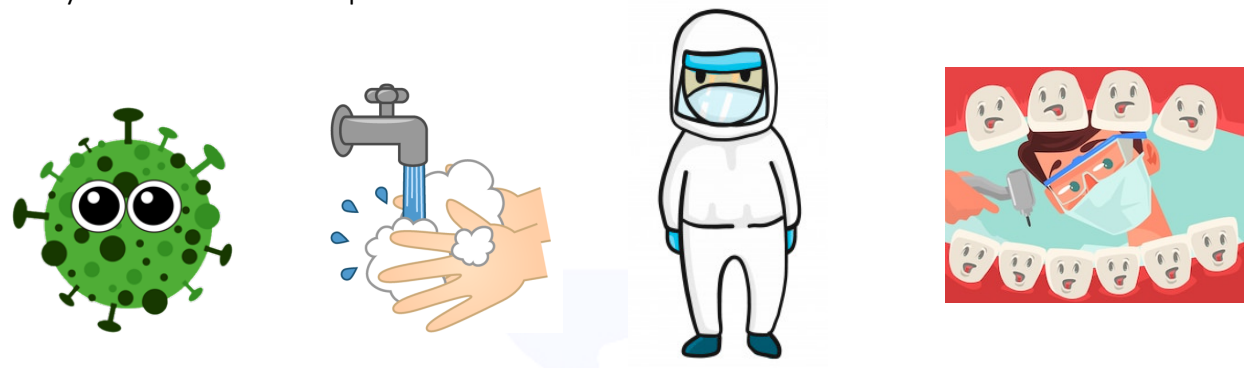

Sin embargo, tu salud bucal es importante y luego de haberte explicado que necesitas realizarte el tratamiento del que hablamos, quiero preguntarte, si comprendiste la información que te di y si estas de acuerdo con realizarlo. Está bien si quieres preguntarle a mamá, a papá o a tu representante legal antes de dar tu respuesta, también puedes hacer todas las preguntas que quieras y las responderé para ti sin que nadie se moleste por ello.

Si aceptas coloca una $\mathrm{X}$ en el cuadro al lado de tu nombre.

Nombre:

Representante: Odontólogo:
Firma:

Firma:

Lugar:
DNI O CC:

DNI O CC:

Fecha:

\section{ALQP ASOCIACIÓN LATINOAMERICANA DE ODONTOPEDIATRÍA}




\section{Anexo 3}

Cod. 608-195

\section{Consentimiento Informado para Tratamiento de Odontopediatría en tiempos de COVID-19.}

YO, con el documento de identidad:

manifiesto que se me ha proporcionado información oportuna, completa, comprensible, clara, precisa, sin tecnicismos y fidedigna en respeto de mis derechos y como expresión de valor de mi autonomía y libertad de decisión y en tal sentido consiento recibir tratamiento odontológico durante la pandemia por la Covid-19. Por lo tanto, entiendo:

- Que el periodo de incubación de este virus es largo y durante el cual sus portadores incluyendo mi persona pueden estar asintomáticos o presentar síntomas leves e inespecíficos de la enfermedad, lo que lo hace altamente contagioso.

- Que debido a la limitación para la realización de pruebas virales y el tiempo de espera para su resultado, es difícil determinar quién es portador del virus y quién no al momento de la consulta.

- Que los procedimientos odontológicos pueden generar aerosoles que permiten la diseminación de la enfermedad. La naturaleza ultrafina del aerosol que producen los equipos odontológicos le permite permanecer suspendido en el aire por minutos o hasta horas, pudiéndose transmitir el virus.

Afirmo que:

- He suministrado información veraz sobre mi estado de salud y he respondido con sinceridad las preguntas en relación con este aspecto que me ha formulado el especialista del consultorio (iniciales).

- Confirmo que no he presentado, en los últimos 14 días, ninguno de los síntomas de la siguiente lista: fiebre, dificultad respiratoria, tos seca, secreción nasal, dolor de garganta, ni hemos estado en contacto con personas con confirmación de COVID-19 o con cuadro respiratorio agudo en las últimas dos semanas (iniciales)

- Se me ha dado información pertinente sobre los protocolos de bioseguridad implementados en el consultorio dental para minimizar el riesgo de contagio y ofrecer un entorno lo más seguro posible.

- Comprendo que a pesar de todos los cuidados, la eventual emisión de aerosoles y la proximidad entre el paciente y el equipo odontológico, así como la realización de procedimientos en la cavidad bucal, hacen que el riesgo cero no se pueda garantizar (iniciales)

- Comprendo que se me ha suministrado información completa sobre el diagnóstico, plan de tratamiento, objetivos y alternativas de tratamiento y que debido a la contingencia actual las opciones terapéuticas minimizan o eliminan procedimientos generadores de aerosoles, siendo mínimamente invasivas para el paciente y están sustentadas en la literatura científica.

(iniciales)

- Luego de suministrada toda la información, de que se me aclararan satisfactoriamente las preguntas que realicé y de que se me permitiera analizar sin presión, ni coacción los riesgos y beneficios del tratamiento odontológico que se me ha propuesto, en pleno uso de mis facultades y haciendo valor a mi autonomía, decido consentir la realización del mismo o rechazarlo

Nombre:

Odontólogo Tratante:
Firma:

Firma:

Lugar:
DNI O CC:

DNI O CC:

Fecha:

\section{ALQP ASOCIACIÓN LATINOAMERICANA DE ODONTOPEDIATRÍA}




\section{Anexo 4}

Cod. 608-192

\section{Consentimiento Informado para Investigación en Odontopediatría en tiempos de COVID-19.}

YO,

tutor legal del paciente: con el documento de identidad:

información manifiesto que se me ha proporcionado mis derechos y de los de mi representado y como expresión de valor de mi autonomía y libertad de decisión y en tal sentido consiento que mi representado participe en la investigación titulada:

la cual se está realizando durante la pandemia por la Covid-19. Por lo tanto, afirmo que:

- He suministrado información veraz sobre mi estado de salud y el de mi representado y he respondido con sinceridad las preguntas en relación con este aspecto que me ha formulado el investigador o grupo de investigadores.

- Confirmo que ni mi representado ni yo hemos presentado, en los últimos 14 días, ninguno de los síntomas de la siguiente lista: fiebre, dificultad respiratoria, tos seca, secreción nasal, dolor de garganta, ni hemos estado en contacto con personas con confirmación de COVID-19 o con cuadro respiratorio agudo en las últimas dos semanas.

- Tengo conocimiento de los protocolos de bioseguridad implementados para llevar a cabo la investigación, permitiendo minimizar el riesgo de contagio y ofrecer un entorno lo más seguro posible. Sin embargo, comprendo que a pesar de todos los cuidados, la eventual emisión de aerosoles y la proximidad entre el paciente y el equipo odontológico, así como la realización de procedimientos en la cavidad bucal, hacen que el riesgo cero no se pueda garantizar.

- Se me ha suministrado información suficiente sobre el objetivo que persigue la investigación en la que mi representado participará, así como los procedimientos a seguir, los beneficios y riesgos inminentes y se me ha especificado si mi representado recibirá alguna compensación por su participación.

- Entiendo que la investigación que se está realizando está destinada a generar nuevos conocimientos en odontopediatría y sus resultados buscan el máximo beneficio de la población pediátrica.

- Se me ha explicado que el manejo de los datos obtenidos será confidencial y se resguardará en todo momento la identidad de mi representado y que al finalizar la investigación se me proporcionará información sobre los resultados obtenidos.

- Luego de suministrada toda la información, de que se me aclararan satisfactoriamente las preguntas que realicé y de que se me permitiera analizar sin presión, los riesgos y beneficios de la participación de mi representado en la investigación, en pleno uso de mis facultades y haciendo valor a mi autonomía, consiento la participación de mi representado y el mismo es voluntario, sin coacción ni manipulación alguna, haciendo valer mi autonomía y libertad de decisión, también entiendo que puedo interrumpir su participación en cualquier momento sin perjuicios, represalias o pérdida de los derechos de mi representado.

Nombre:

Investigador responsable:
Firma:

Firma:

Lugar:
DNI O CC:

DNI O CC:

Fecha:

\section{ALQP ASOCIACIÓN LATINOAMERICANA DE ODONTOPEDIATRÍA}




\section{Anexo 5}

Cod. 608-193

\section{Asentimiento Informado para Investigación en Odontopediatría en tiempos de COVID-19.}

Hola! Soy tu odontopediatra y estoy haciendo un estudio que consiste en Se que en los últimos meses las cosas han cambiado mucho y ahora debemos protegernos los unos a los otros usando tapabocas (mascarilla), lavando nuestras manos constantemente con agua y jabón y en mi caso, debo usar un uniforme nuevo que me ayuda a resguardarme a mi y a ti mientras hago mi trabajo, eso es porque existe un virus nuevo llamado coronavirus que produce una enfermedad llamada Covid-19 que nos puede enfermar y debemos evitar que eso ocurra.

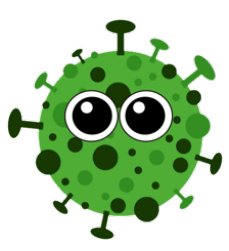

Realizar

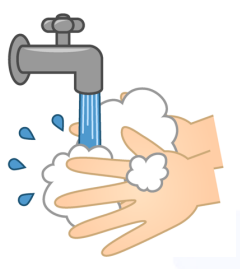

estudio

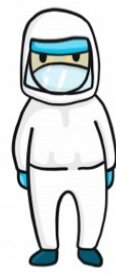

importante

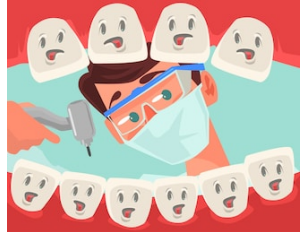

porque

permitirá y luego

de conversar contigo sobre la investigación y comentarte los beneficios y los posibles riesgos, quiero preguntarte, si comprendiste la información que te di y si estas de acuerdo con participar. Está bien si quieres preguntarle a mamá, a papá o a tu representante legal antes de dar tu respuesta, también puedes hacer todas las preguntas que quieras y las responderé para ti sin que nadie se moleste por ello.

Si aceptas participar coloca una $\mathrm{X}$ en el cuadro al lado de tu nombre.

Nombre:

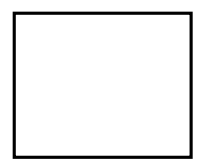

Representante:

Firma:

Firma:

Lugar:
DNI O CC:

DNI O CC:

Fecha:

\section{ALQP ASOCIACIÓN LATINOAMERICANA DE ODONTOPEDIATRÍA}




\section{Anexo 6}

Cod. 608-196

\section{Consentimiento Informado para Investigación en Odontopediatría en tiempos de COVID-19.}

YO, con el documento de identidad:

manifiesto que se me ha proporcionado información oportuna, completa, comprensible, clara, precisa, sin tecnicismos y fidedigna en respeto de mis derechos y como expresión de valor de mi autonomía y libertad de decisión y en tal sentido consiento participar en la investigación titulada:

la cual se está realizando durante la pandemia por la Covid-19. Por lo tanto, afirmo que:

- He suministrado información veraz sobre mi estado de salud y he respondido con sinceridad las preguntas en relación con este aspecto que me ha formulado el investigador o grupo de investigadores.

- Confirmo que no he presentado en los últimos 14 días, ninguno de los síntomas de la siguiente lista: fiebre, dificultad respiratoria, tos seca, secreción nasal, dolor de garganta, ni hemos estado en contacto con personas con confirmación de COVID-19 o con cuadro respiratorio agudo en las últimas dos semanas.

- Tengo conocimiento de los protocolos de bioseguridad implementados para llevar a cabo la investigación, permitiendo minimizar el riesgo de contagio y ofrecer un entorno lo más seguro posible. Sin embargo, comprendo que, a pesar de todos los cuidados, la eventual emisión de aerosoles y la proximidad entre el paciente y el equipo odontológico, así como la realización de procedimientos en la cavidad bucal, hacen que el riesgo cero no se pueda garantizar.

- Se me ha suministrado información suficiente sobre el objetivo que persigue la investigación en la que participaré, así como los procedimientos a seguir, los beneficios y riesgos inminentes y se me ha especificado si recibiré alguna compensación por mi participación.

- $\quad$ Entiendo que la investigación que se está realizando está destinada a generar nuevos conocimientos en odontopediatría y sus resultados buscan el máximo beneficio de la población pediátrica.

- Se me ha explicado que el manejo de los datos obtenidos será confidencial y se resguardará en todo momento mi identidad y que al finalizar la investigación se me proporcionará información sobre los resultados obtenidos.

- Luego de suministrada toda la información, de que se me aclararan satisfactoriamente las preguntas que realicé y de que se me permitiera analizar sin presión, los riesgos y beneficios de mi participación en la investigación, en pleno uso de mis facultades y haciendo valor a mi autonomía, consiento mi participación y la misma es voluntaria, sin coacción ni manipulación alguna, haciendo valer mi autonomía y libertad de decisión, también entiendo que puedo interrumpir mi participación en cualquier momento sin perjuicios, represalias o pérdida de mis derechos.

Nombre:

Investigador responsable:
Firma:

Firma:

Lugar:
DNI O CC:

DNI O CC:

Fecha:

\section{ALQP ASOCIACIÓN LATINOAMERICANA DE ODONTOPEDIATRÍA}




\section{Bioethical framework for the pediatric dental practice in the times of COVID-19}

\section{Introduction}

In early 2020, a new virus that causes severe acute respiratory syndrome (SARS-CoV-2) spread globally causing the coronavirus disease or COVID-19. The rate of new infections varies by day and can be monitored in real time. ${ }^{1}$ With respect to the current situation in Latin America, confirmed cases of COVID-19 have increased significantly. ${ }^{2}$ The World Health Organization (WHO) has declared this situation a pandemic due to the alarming levels of spread of the disease and especially due to the complications that can arise after infection. ${ }^{3}$

Since the beginning of the global health emergency, efforts in Latin America to stop the spread of the disease have been negatively affected due to the previous continent's context before the pandemic, and it includes: poverty, inequality, discrimination, the structural weakness of public health systems and in some countries, political and institutional instability. These factors have made difficult the efficacy of confinement and social distancing measures, as well as other actions in accordance with the International Health Regulations (IHR), the recommendations issued by the World Health Organization (WHO), and the Pan American Health Organization (PAHO).

As a result of this situation, the population of the different countries at the American continent has continued to be extremely affected by the global pandemic. Currently there is a large social vulnerable group with COVID-19, requiring priority care and requesting the defense and protection of their rights as human beings. People with COVID-19 run a special risk of transgression of their human rights, in particular their rights to Life and Health, for which it is necessary an adequate provision of health and medical facilities, goods and services to carry out the diagnosis and treatment. ${ }^{4}$

Dentists, hygienists, dental assistants and patients have always had a high risk of cross infection due to theirexposureto pathogenic microorganisms at the oral cavity and in the respiratory tract. The environment of the dental practices involves dangerously high levels of microorganisms as a result of close contact with the patient's oral cavity and the presence of these in the aerosols generated during some treatments. ${ }^{5}$

The urgent call made by the different health sectors to prioritize the bioethical approach in all aspects related to clinical care and even research during the current public health crisis, has required the collaborative work of different academic and clinical groups that contribute to provide a bioethical response to pediatric dentistry care in this time of pandemic caused by COVID-19.

The Latin American Pediatric Dentistry Association (ALOP) has successfully convened a multidisciplinary team to build a bioethical framework that allows a better understanding of decision-making by 
promoting approaches based on different levels of evidence and supported by ethics. Thus, this paper has been designed to initiate an approach about the debate related to the ethical concerns that derive from dental care and the potentially critical standards during the current pandemic contingency. Within this framework, pediatric dentists will be able to reinforce some important concepts, including the three main duties of medical and dental care during a public health emergency: planning, safeguarding, and guiding. This paper also seeks to provide highlights that can guide the different Ethics Committees at health and academic institutions, to support health professionals who care for patients during the contingency.

The objectives of this bioethical framework are:

1. Highlight the importance of the informed and assent consent and its use for the performance of dental treatments in the pediatric population, as well as for research in times of COVID-19.

2. Implement a clear and concise guide that provides and encourages the bioethics reflection by pediatric dentists during dental care in times of COVID-19.

Informed consent and assent in dental pediatrics in times of COVID-19. Quarantine / confinement period descaling period

Free, prior, full, and informed consent derives from recognized rights by the inter-American system, such as the right to health, the right to receive and have access to adequate information, as well as not to be subject of arbitrary interference in private life. Likewise, it is a central aspect in the development of human rights' bioethics, understood as a valuable tool to frame, and solve the problems and dilemmas related to the pandemic. ${ }^{4}$

There is an ethical duty within the universal principle of autonomy, which is to respect the patient's rights of self-determination and confidentiality. This ethical duty can be particularly challenging with the current pandemic, since it is necessary to act within the acceptable treatment and this depends on the health situation of each country and region. In the current context, public health circumstances force the pediatric dentist to postpone certain treatments, especially elective treatments, until the situation improves or when the confinement is lifted.

Any dental treatment must obtain the prior, free and informed consent of the patient's legal representative. That consent, which preferably must be obtained in writing, must provide timely, complete, understandable, clear, reliable and in nontechnical language, taking into account their particularities and specific needs. In the same way, the legal guardians or caregivers must be informed about the diagnosis, the proposed treatment, the objective of the treatment, the programmable treatment time, the expected benefits and risks of the treatment, the possible unfavorable effects of the proposed therapy, the alternative treatments including the less intrusive, the side effects of other alternative treatments, the possible pain or discomfort to be suffered by the patient, among others. ${ }^{4}$

To obtain an informed consent from the legal guardian of a pediatric patient under the ethical principle of autonomy in times of COVID-19, the caregiver has to receive clear and precise information about the 
risks of the viral infection for the patient, their family and the community. Knowing the risks, the caregiver can accurately assess the risks and benefits to be gained from the pediatric dental treatment, depending on the individual circumstances of the patient. It is important to differentiate and prioritize those procedures that can be considered emergencies or dental emergencies, from all those defined as non-emergency treatments. ${ }^{6}$

Regarding the confidentiality of patient clinical records, the pediatric patients and their legal guardians must be informed about the possible legal requirements to share information in case of suspected contagion within the office. Otherwise the dentist and his staff must continue to maintain and respecting the confidentiality and privacy of all patients. ${ }^{6}$

It is important to highlight the importance of preserving the privacy and protection of personal data of people with COVID-19, especially in these times when the use of digital tools and applications has increased, and including the use of sensitive personal information in the context of the pandemic. There are different regulations and a strong legal framework about data protection, and about the role of regulatory bodies to monitor the compliance on these rights. ${ }^{4}$

In the development of the child as a person, maturity, autonomy and full decisionmaking capacity are acquired through a progressive process for many years. Autonomy allows the person to decide on their individual acts and the reasoning on these acts. Therefore, autonomy involves two ethical precepts: all people must be treated as autonomous individuals and all people with decreased autonomy have the right to be protected. Children achieve full identification of their individual rights and universal ethical principles around the age of 14; however, age is not the optimal measure of mental capacity.

Children, adolescents and other vulnerable populations may have impaired decisionmaking capacity, and they are sometimes not empowered to consent themselves. The responsible adults will determine if the risk and benefit ratio is acceptable to allow the performance of a certain procedure, especially in the case of invasive procedures, granting or not consent for its performance. The children must be explained the procedure, in an understandable way to them, and posteriorly, the dental pediatrician will be able to request the informed consent.

Between the ages of 7 and 18, an intermediate situation arises between the delegated consent to a legal guardian and the autonomous consent, which is called assent. To achieve this assent, the child or adolescent has to receive enough information about the procedures to be carried to him/her and adapt theinformation according to her/him age. Depriving him or her, due to a misunderstood attitude of paternalism, is immoral because it annuls him/her as a person. Asking and counting with the child is a way to recognize her/ him dignity as a person and his/her condition as a moral subject. This context is not only according to ethical criteria, but also a legal criterion. Assent is an act of acceptance with no legal value that must always be accompanied by a verbal or preferably written consent signed by the minor's parents or guardians. It implies recognition of autonomy and constitutes an act of great ethical value, and it is sought that the consent of the parents does not 
exclude the consent of the children. When an agreement between the parents and the minor is in doubt, the consent should not be signed until a dialogue between the minor, her parents and the pediatric dentist has been made, and when the child expresses the will to agree with the consent and with the treatment to be developed. 4,6

This document proposes generic models for informed consent, as well as informed assent, for the performance of pediatric dentistry treatment during the COVID-19 pandemic. (Annexes 1-3)

\section{Ethical aspects in the clinical care of pediatric dental patients in the times of COVID-19}

The ethical problems inherent to preventive and curative care in pediatric dentistry are numerous and frequent. Nevertheless, in times of epidemics and pandemics such as the currently COVID-19 pandemic, they can carry a significant burden on the health of the pediatric population and lead to significant social and economic disorganization. In addition to this, some ethical dilemmas are accentuated due to the biological risk associated with dental care. However, the moral duty indicates that timely care must be given to all patients who require it. In this sense, it is important to become aware of these ethical dilemmas and of the individual and social values that constitute the basis of civic and professional action, as well as the implemented local and regional health policies.

The American Dental Association ${ }^{7}$ (ADA) recommends keep using the Principles of Ethics and the Code of Professional Conduct (Code of Ethics) as a guide in patient care during the COVID-19 pandemic. The ethical principles of autonomy, non-maleficence, beneficence, justice and truthfulness must be maintained as fundamental pillars of professional activity. The general premises of the Code of Ethics of doing no harm and putting the welfare of the patient first are maintained. Government recommendations and announcements are often consistent with the Code of Ethics, however, they may require dentists to take additional measures beyond those normally required and must be followed according to national and regional regulations.

\section{The value of life and health}

All human life is equally valuable and dignified, and everyone equally has the right to life and health. The pediatric dentist must provide quality dental care, with all the resources that minimize the biological risks derived from it, in such a way as to guarantee the success of the procedure performed and that the benefits outweigh the possible risks that could arise with the care.

Patient care during the pandemic must be guided by respect for personal dignity. It is an essential and intrinsic value that allows us to consider every human being as an end in itself, never as a means, a carrier of a singularity and essential value, which transforms them into a unique and unrepeatable being. Among other applications, respect for dignity prevents considering criteria for the allocation of resources that are influenced by issues outside the personal essence, such as age, social value, and disability, among others. The importance of quality and meaning of life for each of the patients should be emphasized. 
To delve deeper into the value of life and health requires the application of ethical principles that are mentioned below: ${ }^{8}$

1. Freedom: is part of human rights, which are universal legal guarantees that protect individuals or groups against actions that interfere with their individual rights and against human dignity. All actions taken to control an epidemic or pandemic, including medical and dental treatments, must be carried out with the utmost respect for human rights.

2. Justice: the pediatric dentist must pay equal care to all, without discrimination of age, gender, race, social and economic condition. This ethical principle requires that the risks, benefits, and burdens of health actions be distributed fairly. "It is the fair, equitable and appropriate distribution in society determined by justified norms that structure the terms of social cooperation." The oral health of the pediatric population must be protected.

3. Equity: the distribution of resources must be done according to needs and urgencies and this must prevail over the economic resources of the different groups or parts of a society. However, it is worth noting that priority should be given in dental care to those with the greatest severity or oral conditions or the most vulnerable. Lack of equity refers to differences in health that are unnecessary, avoidable, and that are considered unfair or illegitimate.

4. Solidarity: It represents the responsibility and shared union between people. Individuals with mutual solidarity share common interests and are not separated by opinions, purposes, or actions. Solidarity is closely related to the principles of justice and equity and represents a social obligation that leads to favor the care of the most vulnerable in a society, rather than as a personal attitude. Solidarity should be the guiding principle of public health, in general and with greater emphasis, in the approach to the pandemic. Solidarity is presented as a barrier against social inequality and discrimination. In the care of the pandemic by COVID-19, it takes on special significance since its victims increase in the same way that the frailties, social inequities, vulnerabilities and stigma increase. The true value of solidarity is expressed when the person who receives the gesture of solidarity lacks all power, such as those who suffer the harmful consequences of the pandemic. There the authentic humanitarian sense of brotherhood is reflected.

5. Reciprocity: A relationship between parties is characterized by a corresponding mutual action.

6. Professional Responsibility: Ethically it means taking charge of what is done in front of the affected person, but it also means taking care of another vulnerable human being.

In this context, the ethical values to rationalize and distribute resources in times of pandemic converge in four fundamental values:

- Maximize the benefits that can be produced during dental care using the least amount of resources or those with the least biological risk.

- The benefits that can be produced 
during dental care using the least amount of resources or those with the least biological risk.

- Do not discriminate. Treat all patients with the same respect and dedication.

- Promote and reward the work of health personnel during the pandemic.

- Give priority to the most serious situations and the sickest. ${ }^{8}$

During public health emergencies the clinical practitioners, and in this case the pediatric dentists, must balance several duties, between the patient-centered care, the scope of clinical ethics, and the promotion of equality of persons and equity in distribution of risks and benefits in society. ${ }^{9}$

The majority of clinical practitioners in the world have been trained focused on patientcentered practice, and only a small percent of them have been trained on a patient care guided by public health considerations. ${ }^{10}$ This new context, where the pandemic shifted the way to distribute human and economic recourses, has created a great tension especially in clinical teams located in developing countries, that continuous face barriers of access in healthcare, and limited resources, and more now during this public health crisis. ${ }^{11,12}$

Traditionally, clinical care is patientcentered, so it is based on the preferences and values of each patient. Aversely, when the health professionals are working in the context of a public health emergency such as the current one, normal care is interrupted and the health of the population is promoted by minimizing morbidity and mortality through the prudent use of resources and strategies. ${ }^{13,14}$

The main objective of professional practice should be to act ethically, providing a high level of care, limiting uncertainty or anxiety about how to proceed, regardless of whether it takes place under normal or emergency circumstances. ${ }^{9}$ In the context of the pandemic, protocols should be established to prioritize patients based on the need for required treatment, and based on possible resource limitations. ${ }^{11,16}$

The ethical duties that apply to the COVID-19 pandemic and that must be balanced are: the duty of medical care that requires fidelity to the patient, relief of suffering and respect for the rights and preferences of patients, the duty of care and its ramifications as the main focus of clinical ethics and the duty to promote equity for the care of people, ensuring an adequate distribution of benefits and risks in society. These ethical duties create important divisions such as promoting public safety, protecting community health, and allocating limited resources, among others. Both the duties mentioned and their ramifications are the main focus of public health ethics. ${ }^{11,17}$

It is the duty of the health professionals to know how to plan and manage the uncertainty that may arise from handling foreseeable and emerging ethical challenges during the pandemic on how to "do the right thing" in clinical practice when duties and values conflict. These challenges often affect the strength of health personnel and make them confront themselves. Planning for ethical challenges in a public health crisis like the current one includes identifying potential triage decisions, tools and processes, staff shortages, changes in space, and difficulties related to declining supplies. ${ }^{17}$

In public health emergencies, the main call is to respond to the greatest demand for 
care with the least amount of resources. Routine therapeutic behaviors may need to be modified, adapting care according to infection control protocols, rational use of resources, and availability of personnel, space, and supplies. However, bioethical considerations emphasize that the critical patient awaiting specialized dental care should be the first to receive dental care. ${ }^{11,12}$

During the public health crisis of COVID-19, it is necessary to safeguard all vulnerable people including health workers and people susceptible to further complications derived from the disease due to factors such as age, underlying health conditions and those with pre-existing barriers accessing to medical care. ${ }^{11}$ In the case of dentistry, all dental personnel must be recognized as a vulnerable and highrisk population.

Monitoring and doing the follow up of patients, particularly the most vulnerable, takes special importance during the pandemic, complying with professional responsibility. The use of a remote care tools, such all those used in teledentistry, allows the clinicians to maintain contact with the patients, carry out health promotion activities, guiding and allowing the best possible triage to be carried out, and showing solidarity to determine the cases that should be attended in person. ${ }^{18}$

Institutional Ethics Committees can support health professionals, patients and family members in making informed decisions, based on both the rights of patients and professional responsibility in health, considering the four principles of bioethics: autonomy, justice, beneficence and non-maleficence. ${ }^{15}$ Equally, health professionals must prepare for service during public health emergencies, recognizing the importance of promoting the equality of people and equity in the distribution of benefits and risks for society, supporting in the best way clinical practice during the pandemic, focused on patient care, management with small staff, and developing strategies to address staff moral distress under crisis conditions. ${ }^{15,19-21}$

\section{Legal framework}

The purposes of any health care or service are the protection of life and health, both physical and mental, the optimization of well-being in a comprehensive manner, non-abandonment, respect for dignity as a human being and self-determination, using the maximum of the available resources for a better treatment according to the possibilities.

For the protection of vulnerable populations, the countries must guide the measures adopted under the principles of equality and non-discrimination in accordance with Inter-American and International Human Rights standards. In this sense, the Unesco Declaration on Bioethics and Human Rights is relevant, as well as the fundamental aspects of public health ethics recognized by national and international specialized institutions on the area. Also has valuable relevance the WHO and PAHO technical guidelines on the virus, as well as the pronouncements of the National Bioethics Committees, scientific and medical societies and other medical bodies.

Each professionalis responsibleforknowing and abiding by the laws, regulations and standards that govern the ethical aspects of dental care in their locality. 
For the protection of the health rights for the pediatric population, it is necessary to recognize and guarantee the right they have to receive adequate health services; these must be immersed in a regulatory framework in health care and treatment protocols with clear parameters of care. Likewise, these must be reviewed and updated, according to the best scientific evidence. $^{4}$

The International Dental Federation (FDI) ${ }^{22}$ published important recommendations to consider for dental practice and the promotion of oral health during the coronavirus pandemic. In this framework, the FDI established some fundamental principles:

- Oral health is an essential public service, fundamental for well-being and health in general and must be accessible to the entire population, therefore, the corresponding authorities must guarantee its access.

- Dental professionals must be part in the debates and decision-making related to the regulation and orientation of medical care during the pandemic.

- Authorities should provide the necessary financial and administrative support to dentists to reduce the financial burden on dental practices and ensure the continuation of adequate care during and after COVID-19.

- All regulations and guidelines directed towards dental professionals must take into account the situation and the public health needs of each country and not only the individual risk of infection of professionals and patients.

- Dentists must strictly follow all current national guidelines and regulations, including those related to Personal Protective Equipment (PPE), procedures for different treatments and patient admission protocols. The authorities must guarantee access to PPEs at affordable prices.

- Dental professionals have the responsibility to ensure emergency care, whenever possible.

- It is a duty to use every opportunity to reinforce oral cavity disease prevention messages in order to reduce the need for treatments, elective consultations, and costs for medical care.

- Dentists have a responsibility to protect the health and well-being of their staff and patients.

- Research on COVID-19 and its transmission, including specific considerations for dental practice, are necessary to allow adequate guidance in this area.

\section{About the dental treatment in children and youth during the COVID-19 pandemic}

The incubation period of this virus varies between individuals and can be from 2 to 14 days, where children may be asymptomatic or with mild and nonspecific symptoms of the disease. Therefore, pediatric patients and their parents or guardians should be considered potential carriers of the virus unless proven otherwise. The transmission of the virus is known and has been expanded in previous publications belonging to the ALOP COVID-19 Resource Center, however, it is always important to highlight that the pediatric dentist as health 
personnel is exposed to situations whose risk of contagion is potentially high. ${ }^{23}$

A bioethical vision of the current pandemic context, promote the performance of dental treatments in the pediatric population when required, constituting an ethical duty and a responsibility of the dentist. It requires providing high quality care, but taking the enough measures to decrease the collective biological risks.

The treatments must be carried out considering the life and general health of the patients, including their oral health, as a priority, so emergency treatment in pediatric dentistry will always be justified. However, it is the duty and responsibility of the dentists as health professionals at this historical moment, to ensure compliance with international biosafety protocols within their dental practices.

Another aspect to highlight is the importance of an efficient practice simplifying the procedures to reduce the generation of aerosols and thus reduce the risk of contagion by cross infection, respecting and honoring the principle of beneficence and non-maleficence. Treatment options now exist that minimize or eliminate aerosol-generating procedures, many of which are based on contemporary karyology, are well documented in the scientific literature, and are minimally invasive in nature. ${ }^{24}$

The clinical guidance can never cover all possiblecircumstances, and the professional and social responsibility must be exercised taking decisions about to provide or not certain treatments for pediatric patients. It is important that the pediatric dentist follow local, national, and regional guidelines, and during the COVID-19 pandemic, it is important to maximize infection control in defense of everyone's health. Once the restrictions on dental practice begin to be lifted, it is recommended that procedures that generate the least amount of aerosols and are minimally invasive continue to be performed. These include atraumatic restorative treatment (ART), sealing of carious lesions with pit and fissure sealant, the use of silver diamine fluoride, selective caries removal and the Hall technique. ${ }^{24}$

It must be taken into account that some countries are progressively adopting transitions towards the full reactivation of activities, making it necessary to adopt the necessary measures to minimize the risks of contagion, prevent massive outbreaks of COVID-19. This includes public policies ensuring the availability of diagnostic tests, improving accessibility to the health services necessary to ensure that, should there be an increase in cases, medical resources, hospital infrastructure and the availability of supplies are available. ${ }^{4}$

\section{Biosecurity considerations}

Since the transmission of the infection occurs by droplets suspended in the air, this being the main route of spread of the virus, it is recommended that the work team use all the barrier measures recommended by international entities, namely: glasses and protective mask, face mask N95 or similar, gloves, cap and suitable protective clothing for all dental care provided during the epidemic period. ${ }^{25}$

\section{Research in children and youth in times of pandemic}

In accordance with international ethical standards for research in the area of 
human health, ${ }^{25-27}$ the participation of children and adolescents is essential for the research of diseases or pathological conditions, to which this age group is particularly susceptible and the studies must be channeled in the work "with" children and not "in" children. Based in the modern concept of child-centered research, this approach considers the opinion and decision of children to participate in the research process. In this context, the child's participation should be active throughout the investigation, their opinion should be taken seriously and depending on age, they can be competent and thoughtful in reporting their own experiences.

Research in dentistry is essential to provide the best clinical treatment to children and adolescents, which must be based on solid and available scientific evidence on the therapeutic efficacy of the different procedures, as well as the various dental materials that will be used and the safety of the same in this population. ${ }^{6}$

Research can be considered scientific when it is based on a series of values that arise from the same nature of science, whose purpose is the search for objective truth. It is important to talk about an ethic research, that is, a conscientious research, where whose results are used correctly, especially when the population to be studied is pediatric. ${ }^{28}$

The researcher has a main responsibility to guarantee a study with quality, in terms of originality, relevance and value for their area of knowledge, as well as highlighting the importance of considering the ethical aspects concerning the research. All research activities must be characterized by honesty (from the initial approach, the study design, to the dissemination and publication of the results) and to achieve this goal, the researcher or group of researchers must be honest.

An ethics committee does not replace the morality of the researcher, which is reflected in multiple aspects and moments of the process: the careful and methodical collection of data, the meticulous data keeping, and the realistic interpretation of the results, without forcing or adjust the data to the hypotheses that interest the researcher, etc.

The first thing to keep in mind in dental research in children and adolescents is that scientific truth is at the service of the person and not the other way around. Therefore, the truth in medicine is not an end in itself, but a means at the service of man, which is considered an end per se. This should not mean an obstacle or a deterrent to the advancement of knowledge through the scientific method, but rather, it ensures quality and well-prepared research, in accordance with its ultimate purpose of improving the quality of life of the human beings with respect of their dignity. ${ }^{29}$

The protection of the dignity of the human being under investigation must also be taken into account, counting on their free and well-informed consent about the risks and possible consequences of the procedures carried out on their person. ${ }^{29}$

Research ethics emphasizes on the responsibility in the exercise of scientific activity, which includes avoiding unnecessary suffering to subjects participating in research. This responsibility also includes fostering in a special way the ethical reflection that allows developing the incredible potential of scientific research, in order to improve living conditions in the future and not to deteriorate them. ${ }^{29}$ 
Finally, full transparency in the investigation must be guaranteed. It is the responsibility of the researcher to always be able to give an account of their work, thus respecting, on the one hand, the opinion of the scientific community when an evaluation of the discoveries has made, and on the other hand, being aware of the social impact and responsibility towards society that it has scientific activity. ${ }^{29}$

Due to the exceptional health crisis that is currently being witnessed due to the spread of COVID-19, it imposes an ethical duty to do research in order to improve prevention and health care for the world population. This research is essential to understand the disease, its mechanisms of contagion and expansion, in order to formulate interventions, the better practices that are efficient and effective, and the process that allow to contain the contagion. Similarly, research in this area also makes it possible to evaluate the safety and validity of diagnostic tests, treatments, vaccines, and management strategies. In general terms, the situation does not justify non-observance of the established guidelines, guides and recommendations of current international organizations, which must be respected in all cases. For this reason, it is essential that research is promoted without removing rigor from the ethical and methodological evaluation of the studies in order to achieve the ultimate goal, which is the protection of research subjects. In this vein, it is worth highlighting the importance of obtaining informed consent to ensure that the autonomy of all participants is respected and that their decision is voluntary.

To this document we attach base models of informed consent and assent for conducting scientific research in pediatric dentistry during COVID-19. (Annexes 4 - 6).
The particular situation related to the virulence and ease of contagion of COVID-19, contains challenges related to the documentation of the informed consent process. In that order, when the usual mechanisms are not possible, it is necessary to consider alternative ways (such as contacting participants by video call, developing a record of informed consents by audiovisual means or digital images, obtaining oral consents supplemented with confirmation by mail electronic record and entry in the Medical Record with the signature of one or more health professionals). The relevance and acceptance of the variants will depend on the internal regulations of the institution in which the investigation is carried out, on local regulations and with the authorization of the Institutional Ethics Committees. ${ }^{30}$

\section{Recommendations}

The COV ID-19 pandemic raises ethical and legal issues, which include the allocation of resources and access to medical care as a priority. The four universal ethical principles: autonomy, beneficence, nonmaleficence and justice, can help pediatric dentists in decision-making and in the moral reflection that arises from dental care during the pandemic.

The pediatric dentist has the duty to act prioritizing the principle of beneficence, but respecting their dignity and autonomy. During situations like the current one, human dignity is the highest of all values and is independent of social position or usefulness to others. In this sense, the treatments must be provided regardless of age, gender, origin or ethnicity, color, place of residence, social or economic status (principle of justice). 
COVID-19 amplifies the problems of access to health care and problems related to the resource shortage. Therefore, it is ethically justified to reschedule or cancel elective procedures, however, the ethical duty is to provide essential and adequate care, treating patients according to their needs, using the least amount of available resources and decreasing in the possible generation of aerosols that could increase the risk of contagion and the spread of the disease. ${ }^{31}$

\section{Autores}

Development of this article led by Rosa Gabriela Rondón(Venezuela), Gabriel Zambrano(Venezuela), Martha Mutis (Estados Unidos de América), Paola Beltri (España), Olga Cortés Lillo (España), on behalf of the COVID-19 Interdisciplinary team, LatinAmerican Pediatric Dentistry Association (Asociación Latinoamericana de Odontopediatría).

COVID-19 Interdisciplinary team, Latin-American Pediatric Dentistry Association (Asociación Latinoamericana de Odontopediatría):

JennyAbanto (Brasil), MarianaArmada (Argentina), Paola Beltri (España), Marisol Carrillo Tabakman (Paraguay), Haydée Casaretto (Argentina), Jorge Luis Castillo (Perú), Mónica Gladys Cesetti (Argentina), Bertha Angélica Chávez González (Perú), Ana Claudia Rodrigues Chibinski (Brasil),
Salomon Alberto Cohen (Argentina), Olga Cortés Lillo (España), Luzia Ana Da Silva de Carballo (Venezuela), Gonzalo De la Fuente Alvarez (Chile), Renée Di Nallo (Argentina), María Débora Elizabeth Dricas (Argentina), Sandra Echevarria (Brasil), Piedad Cecilia Echeverry Marin (Colombia), Laura Fedelli (Argentina), Carlos Flores-MIr (Canadá), Andrea Virgina González Carfora (Chile), Lina María Hernández Salas (Colombia), Francisco José Hernández Restrepo (Colombia), Maria Teresa Ibañez Rodriguez (Bolivia), José Carlos Pettorossi Imparato (Brasil), Alejandra Lipari Valdés (Chile), Daniela Madrigal López (Costa Rica), Daniela Catalina Martínez Camus (Chile), María Gabriela Martínez Vásquez (Venezuela), A. Carolina Medina Díaz (Venezuela), Kelly Maria Silva Moreira (Brasil), Elías M. Morón (Estados Unidos de América), Martha Mutis (Estados Unidos de América), Camila Palma (Perú), Gladys Mabel Peña (Argentina), Adriana Pistochini (Argentina), Paloma Planells (España), Gabriel Politano (Brasil), Matias RiosErazo (Chile), Adriana Maria Rubiano Pinzon (Colombia), Karla Mayra Rezende (Brasil), Rosa Gabriela Rondón (Venezuela), Gabriela Scagnet (Argentina), Rosemary Sogbe de Agell (España), Marina Tavares Costa Nóbrega (Canadá), Jorgelina Valente (Argentina), Ernesto Venegas De Herrera (República Dominicana), Rosa Helena Wanderley Lacerda (Brasil), Ana Clara Zabala (Argentina), Gabriel Zambrano (Venezuela).

Información completa del Grupo Interdisciplinario COVID-19 de la Asociación Latinoamericana de Odontopediatría

\section{References}

1. Mallineni SK, Innes NP, Raggio DP, Araujo MP, Robertson MD, Jayaraman, J. Coronavirus disease (COVID-19): Characteristics in children and considerations for dentists providing their care. Int J Paediatr Dent. [Internet]: 2020 [Last review: June 2, 2020]; 30: 245-50. Available at: https://doi.org/10.1111/ipd.12653

2. BBC News Mundo. Coronavirus en América Latina: los países de la región donde más está creciendo el número de contagios de covid-19 [Internet]: BBC; Mayo 2020 [Last review: June 2, 2020]. Available at: https://www.bbc.com/mundo/noticias-america-latina-52667117

3. Organización Mundial de la Salud. Brote de enfermedad por coronavirus (COVID-19). [Internet]: Organización Mundial de la Salud; 2020 [Last review: June 2, 2020]. Available at: https:/www.who.int/es/ emergencies/diseases/novel-coronavirus-2019?gclid=Cj0KCQjw9b_4BRCMARIsADMUIyr1ljG9EJJo_sEtfj7TG_qGskQacPg1DxvxrA6_hr6LHJJz43mpAMgaAtU-EALw_wcB

4. Comisión Interamericana de Derechos Humanos. Derechos humanos de las personas con Covid-19 [Internet]: Organización de Estados Americanos; Abril 2020 [Last review: July 20, 2020]. Available at: http:// www.oas.org/es/cidh/decisiones/pdf/Resolucion-4-20-es.pdf 
5. Checchi V, Bellini P, Bencivenni D, Consolo U. COVID-19 dentistry-related aspects: a literature overview. Int Dent J [Internet] 2020 [Last review: June 2, 2020]; doi:10.1111/idj.12601. Available at: https://onlinelibrary.wiley.com/doi/full/10.1111/idj.12601

6. Garrocho-Rangel A, Cerda-Cristerna B, Pozoz-Guillen A. Bioethical Issues in Conducting Pediatric Dentistry Clinical Research.J Clin Pediatr Dent [Internet] 2018; [Last review: June 2, 2020]; 42(2):85-90. Available at: https://jocpd.org/doi/10.17796/1053-4628-42.2.1

7. American Dental Association. Guidance on continuing to practice ethically during COVID-19. [Internet] 2020 [Last review: 25 Junio 2020]; Available at: https://success.ada.org/ /media/CPS/Files/COVID/ ADA_COVID_How_Do_I_Ethically_See_Patients_During_Pandemic.pdF

8. Poleo J. Ética médica y Covid-19 Comisión Ética Sociedad de Médicos. Asociación de Bioética Clínica. Caracas. 2020: 1-37.

9. Institute of Medicine. Crisis Standards of Care: Systems Framework for Catastrophic Disaster Response. [Internet] Washington, DC; 2012 [Last review: June25, 2020]; Available at: http://www.nationalacademies.org/hmd/ /media/Files/Report\%20Files/2012/Crisis-Standards-of-Care/CSC_rb.pdf.

10. Institute of Medicine. Engaging the Public in Critical Disaster Planning and Decision Making. Workshop summary. [Internet] Washington, DC; 2013 [Last review: June 25, 2020]; Available at: http://www. nationalacademies.org/HMD/Reports/2013/Engaging-the-Public-in-Critical-Disaster-Planning-and-Decision-Making.aspx.

11. The Hastings Center. Ethical Framework for Health Care Institutions Responding to Novel Coronavirus SARS-CoV-2 (COVID-19): Guidelines for Institutional Ethics Services Responding to COVID-19. March 16, 2020.

12. Minnesota Department of Health. Minnesota Crisis Standards of Care Framework: Health Care Facility Surge Operations and Crisis Care [Internet] Minnesota; 2020. [Last review: June 25, 2020]; Available at: https://www.health.state.mn.us/communities/ep/surge/crisis/framework_healthcare.pdf.

13. Christian MD, Hawryluck L, Wax RS, Cook T, Lazar NM, Herridge MS, Muller MP, Gowans DR, Fortier W, Burkle FM. Development of a triage protocol for critical care during an influenza pandemic. CMAJ. [Internet] 2006; [Last review: June 2, 2020]; 175(11): 1377-81. Available at: https://www.ncbi.nlm.nih.gov/ pmc/articles/PMC1635763/\#_ffn_sectitle

14. Hick JL, Hanfling D, Wynia MK, Pavia AT. Duty to Plan: Health Care, Crisis Standards of Care, and Novel Coronavirus SARS-CoV-2 [Internet] Washington, DC. NAM PERSPECTIVES. Discussion paper. National Academy of Medicine; 2020 [Last review: June 2, 2020]. Available at: https://doi.org/10.31478/202003b

15. Italian Society for Anesthesia, Analgesia, Resuscitation, and Intensive Care. Clinical Ethics Recommendations for Admission to Intensive Care and for Withdrawing Treatment in Exceptional Conditions of Imbalance between Needs and Available Resources [Internet]. Italia: Italian Society for Anesthesia, Analgesia, Resuscitation, and Intensive Care. 2020 [Last review: June 2, 2020]. Available at: https://www. academia.edu/42213831/English_translation_of_the_Italian_SIAARTI_COVID-19_Clinical_Ethics_Recommendations_for_Resource_Allocation_3_6_20

16. Whelan A, Young G, Catanese VM. Medical Students and Patients with COVID-19: Education and Safety Considerations. American Association of Medical Colleges [Internet]. 2020 [Last Review: June 2, 2020]. Available at: https://www.aamc.org/system/files/2020-03/Role\%20of\%20medical\%20students\%20 and\%20COVID-19-FINAL.pdf.

17. Adams JG, Walls RM. Supporting the Health Care Workforce during the COVID-19 Global Epidemic. JAMA [Internet]. 2020. [Last Review: June 2, 2020]; 323(15):1439-40. Available at: https://jamanetwork. com/journals/jama/fullarticle/2763136

18. Equipo de Trabajo Multidisciplinario de la Revista de Odontopediatría Latinoamericana. Teleodontología: Aplicación a la Odontopediatría durante la pandemia COVID-19. Rev Odontoped Latinoam. 2020; 10, (2), Available at: https://www.revistaodontopediatria.org/ediciones/2020/2/art-3/

19. Institute of Medicine. Crisis Standards of Care: A Toolkit for Indicators and Triggers. Report brief. [Internet] Washington, DC; 2013 [Last review: June 25, 2020]; Available at: http://www.nationalacademies.org/ hmd/ /media/Files/Report\%20Files/2013/CSC-Triggers/CSC-Triggers-RB.pdf

20. Altevogt BM, Stroud C, Hanson SL, Hanfling D, Gostin LO. Guidance for establishing crisis standards of care for use in disaster situations [Internet] Institute of Medicine of the National Academies; 2009. [Last review: June 25, 2020]; Available at: http://www.inovaideas.org/emergency_books/2. 
21. New York State Department of Health, New York State Task Force on Life and the Law. Ventilator Allocation Guidelines. [Internet]. New York; 2015. [Last review: July 2, 2020] Available at: https://www.health. ny.gov/regulations/task_force/reports_publications/docs/ventilator_guidelines.pdf.

22. Consejo General de Colegios de Dentistas de España. La FDI elabora un decálogo sobre Odontología y salud oral durante la pandemia por la Covid-19 [Internet]. España: Consejo Dentistas; Junio 2020 [Last review: July 2, 2020]. Available at: https://www.consejodentistas.es/comunicacion/actualidad-consejo/ notas-de-prensa-consejo/item/1815-la-fdi-elabora-un-decalogo-sobre-odontologia-y-salud-oral-durantela-pandemia-por-la-covid-19.html

23. Peng X, Xu X, Li Y, Cheng L, Zhou X, Ren B. Transmission routes of 2019-nCoV and controls in dental practice. Int J Oral Sci [Internet] 2020 [Last review: June 2, 2020]; 12(9). Available at: https://doi. org/10.1038/s41368-020-0075-9

24. Asociación Latinoamericana de Odontopediatría. Grupo de Trabajo Interdisciplinaro COVID-19. Tratamiento de la enfermedad de caries en época de COVID-19: protocolos clínicos para el control de aerosoles. Rev Latinoamericana Odontoped [Internet] 2020 [Last review: June 2, 2020]; 10(2). Available at: https://www.revistaodontopediatria.org/ediciones/2020/2/art-2/

25. Asociación Latinoamericana de Odontopediatría. Grupo de Trabajo Interdisciplinaro COVID-19. Ruta de atención para procedimientos de Odontología Pediátrica durante la etapa de confinamiento o cuarentena de la pandemia COVID-19. Revista de Odontopediatría Latinoamericana. 2020; 10(2): https://www. revistaodontopediatria.org/ediciones/2020/2/art-1/ .

26. van Delden J, van der Graaf R. Revised CIOMS International Ethical Guidelines for Health-realted Research Involving Humans.J Am Med Assoc [Internet] 2016 [Last reviewo: June 2, 2020]; 317(2): 1-119. Disponible en: https://cioms.ch/wp-content/uploads/2017/01/WEB-CIOMS-EthicalGuidelines.pdf

27. World Medical Association Declaration of Helsinki. Ethical principles for medical research involving human subjects. J Am Med Assoc. [Internet] 2013 [Last Review: June 2, 2020]; 310(20):2191-4. Available at: https://pubmed.ncbi.nlm.nih.gov/24141714/

28. Galan M. Etica de la investigación. Revista Iberoamericana de Educación. 2010;54(4):1-2.

29. Martin S. Aplicación de los principios éticos a la metodología de la investigación. Enferm Cardiol. [Internet] 2020 [Last Review: June 25, 2020]; 2013;1(1-2):27-30. Available at: https://www.researchgate.net/ publication/340495940_Guidelines_for_dental_care_provision_during_the_COVID-19_pandemic.

30. Ministerio de salud GCABA. Recomendaciones del CCE referidas al Consentimiento Informado en investigaciones sobre COVID 19 [Internet] 2020 [Last Review: June 25, 2020]; 10(2). Available at: https:// www.buenosaires.gob.ar/sites/gcaba/files/recomendaciones_del_cce_referidas_al_consentimiento_informado_en_investigaciones_sobre_covid_19.pdf

31. Gurzawska-Comis K, Becker K, Brunello G, Gurzawska A, Schwarz F. Recommendations for Dental Care during COVID-19. J Clin Med [Internet] 2020 [Last Review: 2 Junio 2020]; 9(1833): 1-15. Available at: https://encyclopedia.pub/1722

Received: $15 / 06 / 2020$

Accepted: 30/07/2020

Correspondence: e-mail: editor@revistaodontopediatria.org 